\title{
Cosmological dynamics of a non-minimally coupled bulk scalar field in DGP setup
}

\author{
Kourosh Nozari $^{1}$ and Narges Rashidi ${ }^{2}$ \\ Department of Physics, Islamic Azad University, Sari Branch, Sari, Iran
}

\begin{abstract}
We consider cosmological dynamics of a canonical bulk scalar field, which is coupled non-minimally to 5-dimensional Ricci scalar in a DGP setup. We show that presence of this non-minimally coupled bulk scalar field affects the jump conditions of the original DGP model significantly. Within a superpotential approach, we perform some numerical analysis of the model parameter space and consider bulk-brane energy exchange in this setup. Also we show that the normal, ghost-free branch of the DGP solutions in this case has the potential to realize a self-consistent phantom-like behavior and therefore explains late time acceleration of the universe in a consistent way.
\end{abstract}

PACS: 04.50.-h, 98.80.Cq, 95.36.+x, 04.65.+e

Key Words: Braneworld Cosmology, Induced Gravity, Bulk Scalar Field, Superpotentials, Phantom-like Behavior.

\footnotetext{
${ }^{1}$ knozari@umz.ac.ir

2n.rashidi@umz.ac.ir
} 


\section{Introduction}

According to the recent cosmological observations, our universe is undergoing an accelerating phase of expansion and transition to the accelerated phase has been occurred in the recent cosmological past [1]. The simplest way to describe the accelerated expansion of the universe is to adopt a cosmological constant. However, huge amount of fine-tuning required for its magnitude and other theoretical problems such as unknown origin and lake of dynamics make it unfavorable for cosmologists [2]. So, to explain this remarkable behavior of the universe, many theoretical approaches have been proposed in recent years $[3,4]$.

Other alternative approaches to accommodate dark energy are modification of general relativity by considering additional spatial dimensions [5-8]. Also teleparallel gravity provides a basis for explanation of the late-time accelerated expansion [9]. In the revolutionary braneworld viewpoint, our universe is a 3-brane embedded in an extra dimensional bulk. Standard matter and all interactions are confined on the brane; only graviton and possibly non-standard matter are free to probe the full bulk. One of the various braneworld scenarios, is the model proposed by Dvali, Gabadadze and Porrati (DGP). This setup is based on a modification of the gravitational theory in an induced gravity perspective $[7,8]$. This induced gravity term in the model leads to deviations from the standard 4-dimensional gravity over large distances. In this braneworld scenario, the bulk is considered as empty except for a cosmological constant and the matter fields on the brane are considered as responsible for the evolution on the brane. Although the DGP setup is successful to explain late-time acceleration of the universe expansion in its self-accelerating branch, the model has ghost instability in this branch [10]. Nevertheless, the normal, non-self-accelerating branch is ghostfree and as has been shown, has the potential to realize interesting cosmological implications $[11,12]$. On the other hand, when a higher dimensional embedding space exists, we are free to consider some bulk matter which can certainly influence the cosmological evolution on the brane and can be a major contributor to the dark energy. One of the particular forms of bulk matter is a scalar field [13]. We have studied with details the cosmological dynamics of minimally coupled bulk scalar field in the DGP setup recently $[14,15]$. But, since scalar field can interact with other fields such as the gravitational sector of the theory, in the spirit of braneworld scalar-tensor theories, we can consider a non-minimal coupling (NMC) of the scalar field with intrinsic (Ricci) curvature induced on the brane. We note that generally the introduction of the $\mathrm{NMC}$ is not just a matter of taste. The NMC is instead forced upon us in many situations of physical and cosmological interest. There are compelling reasons to include an explicit non-minimal coupling in the action. For instance, non-minimal coupling arises at the quantum level when quantum corrections to the scalar field theory are considered. Even if for the classical, unperturbed theory this non-minimal coupling vanishes, it is necessary for the renormalizability of the scalar field theory in curved space. In most theories used to describe inflationary scenarios, it turns out that a nonvanishing value of the coupling constant cannot be avoided [16,17]. Generalization of this scalar-tensor extension to braneworld setup with modified induced gravity in the spirit of $f(R)$ theories has been considered recently [18].

In the present work, we consider a scalar field which is non-minimally coupled with 
intrinsic (Ricci) curvature in the bulk of a DGP braneworld scenario. In section 2 we derive the Bulk-brane Einstein's equations and the scalar field's equation of motion. Also, we find the jump conditions on the brane in the presence of this bulk scalar field. In section $\mathbf{3}$ we consider the cosmology of this DGP-inspired model and find the Friedmann equation for cosmological dynamics on the brane. In this section we reduce the original partial differential field equations to an ordinary differential equation. In section $\mathbf{4}$, we use the superpotential method to perform some numerical analysis on the parameter space of the model and discuss on bulk-brane energy exchange. We show that, since we expect the energy leaks of the brane with expansion of the universe, there is some constraints on parameter space of this scenario. In section 5, we study the late time behavior of this model. We show the normal branch of this DGP-inspired model, in the presence of non-minimally coupled bulk scalar field, can explain the late-time cosmic acceleration.

\section{The Setup}

The 5-dimensional action for a DGP-inspired braneworld model in the presence of a nonminimally coupled scalar field in the bulk can be written as follows

$S=\int_{B} d^{5} x \sqrt{-g}\left\{\frac{1}{2 \kappa_{5}^{2}}\left(1-\xi \phi^{2}\right) \mathcal{R}-\frac{1}{2}(\nabla \phi)^{2}-V(\phi)\right\}+\int_{b} d^{4} x \sqrt{-h}\left(\frac{1}{2 \kappa_{4}^{2}} R+\frac{1}{2 \kappa_{5}^{2}}[K]+\mathcal{L}_{b}(\phi)+\mathcal{L}_{b}^{(m)}\right)$,

where $g_{A B}$ is the bulk metric and $h_{A B}$ is the induced metric on the brane. They are related by $h_{A B}=g_{A B}-n_{A} n_{B}$, where $n_{A}$ is the unit vector normal to the 3 -brane and $A, B$ are the five dimensional indices. The Gibbons-Hawking boundary term is included via jump of the trace of the extrinsic curvature $[K]$ in the brane action. Also, $\kappa_{5}^{2}=\frac{8 \pi}{M_{5}^{3}}$, where $M_{5}$ is the fundamental 5-dimensional Planck mass. The brane Lagrangian $\mathcal{L}_{b}(\phi)$ includes the Standard Model fields which are confined to the brane and depends on the bulk scalar field. It should be noticed that ordinary matter (such as dust matter or perfect fluid) described by $\mathcal{L}_{b}^{(m)}$, is confined to the brane located at $y=0$. In this action, the non-minimal coupling of the bulk scalar field and the 5 -dimensional Ricci scalar is represented by the term $\xi \phi^{2} \mathcal{R}$. We note that, the indices "B" and " b" in integral refer to "Bulk" and "brane " respectively.

The Bulk-brane Einstein's equations calculated from action (1) are given by

$$
\frac{1}{\kappa_{5}^{2}}\left(1-\xi \phi^{2}\right)\left(\mathcal{R}_{A B}-\frac{1}{2} g_{A B} \mathcal{R}\right)+\frac{1}{\kappa_{4}^{2}}\left(R_{A B}-\frac{1}{2} h_{A B} R\right) \delta(y)=T_{A B}^{(B)}+T_{A B}^{(b)} \delta(y),
$$

where $T_{A B}^{(B)}$ and $T_{A B}^{(b)}$ are the bulk and brane energy momentum tensor respectively. Also, $\delta(y)$ is the Dirac delta function with support on the brane which we assume to be located at $y=0$, where $y$ is the coordinate of the extra dimension. In our setup, the energy momentum of the bulk is given by following expression

$$
T_{A B}^{(B)}=\nabla_{A} \phi \nabla_{B} \phi-\frac{1}{2} \nabla^{K} \phi \nabla_{K} \phi g_{A B}-V(\phi) g_{A B}
$$




$$
+2 \xi \nabla_{A} \phi \nabla_{B} \phi-2 \xi \nabla^{K} \phi \nabla_{K} \phi g_{A B}+2 \xi \phi \nabla_{A} \nabla_{B} \phi-2 \xi \phi \nabla^{K} \nabla_{K} \phi g_{A B} .
$$

This energy momentum tensor leads to the following bulk energy density and pressure

$$
\begin{gathered}
\rho^{(B)}=2 n^{2} \xi\left(\phi^{\prime 2}+\phi \phi^{\prime \prime}\right)+6 n^{2} \xi\left(\frac{a^{\prime}}{a}\right) \phi \phi^{\prime}-6 \xi\left(\frac{\dot{a}}{a}\right) \phi \dot{\phi} \\
+\frac{1}{2} \dot{\phi}^{2}+\frac{n}{2} \phi^{\prime 2}+n^{2} V(\phi), \\
p^{(B)}=-\frac{k}{a^{2}} \xi \phi^{2}+\frac{2 \xi}{n^{2}}\left[\dot{\phi}^{2}+\phi \ddot{\phi}+\left(2 \frac{\dot{a}}{a}-\frac{\dot{n}}{n}\right) \phi \dot{\phi}\right]-2 \xi\left[\phi^{\prime 2}+\phi \phi^{\prime \prime}+\left(2 \frac{a^{\prime}}{a}+\frac{n^{\prime}}{n}\right) \phi \phi^{\prime}\right] \\
+\frac{1}{2 n^{2}} \dot{\phi}^{2}-\frac{1}{2} \phi^{\prime}-V(\phi) .
\end{gathered}
$$

We note that, the first three terms in equations (4) and (5), show the effect of non-minimal coupling in the bulk and if we set $\xi=0$, these equations simplify to the usual results for minimal case. $T_{A B}^{(b)}$, the total energy momentum of the brane, is defined as

$$
T_{A B}^{(b)}=T_{A B}^{(m)}+T_{A B}^{(\phi)},
$$

where $T_{A B}^{(\phi)}$ and $T_{A B}^{(m)}$ are the energy momentum tensor corresponding to $\mathcal{L}_{b}(\phi)$ and $\mathcal{L}_{b}^{(m)}$ respectively. The scalar field's equation of motion given by

$$
\nabla^{2} \phi-n^{2} \frac{d V}{d \phi}+\frac{n^{2}}{\kappa_{5}^{2}} \xi \phi \mathcal{R}+n^{2} \frac{\sqrt{-h}}{\sqrt{-g}} \frac{d \mathcal{L}_{b}(\phi)}{d \phi}=0 .
$$

The action (1) implies the following jump conditions

$$
\begin{gathered}
{\left[N^{A} \nabla_{A} \phi\right]=\frac{\delta \mathcal{L}_{b}(\phi)}{\delta \phi},} \\
{\left[K_{A B}-K h_{A B}\right]=-\kappa_{4}^{2} T_{A B}^{(b)} .}
\end{gathered}
$$

To formulate cosmological dynamics on the brane, we assume the following line element

$$
d s^{2}=g_{A B} d x^{A} d x^{B}=-n^{2}(y, t) d t^{2}+a^{2}(y, t) \gamma_{i j} d x^{i} d x^{j}+b^{2}(y, t) d y^{2},
$$

where $\gamma_{i j}$ is a maximally symmetric 3 -dimensional metric defined as $\gamma_{i j}=\delta_{i j}+k \frac{x_{i} x_{j}}{1-k r^{2}}$ where $k=-1,0,+1$ parameterizes the spatial curvature and $r^{2}=x_{i} x^{i}$.

Since here we consider homogeneous and isotropic geometries inside the brane, $T_{B}^{A(b)}$ can be expressed quite generally in the following form

$$
T_{B}^{A(b)}=\frac{1}{b} \operatorname{diag}\left(-\rho^{(b)}, p^{(b)}, p^{(b)}, p^{(b)}, 0\right),
$$


where $\rho^{(b)}=\rho^{(m)}+\rho^{(\phi)}$. Note that here $\rho^{(\phi)}$ is the energy density corresponding to $T_{A B}^{(\phi)}$ and $\rho^{(m)}$ is the energy density corresponding to $T_{A B}^{(m)}$. The extrinsic curvature tensor in the background metric (10) is given by

$$
K_{B}^{A}=\operatorname{diag}\left(\frac{n^{\prime}}{n b}, \frac{a^{\prime}}{a b} \delta_{j}^{i}, 0\right) .
$$

By using the metric ansatz (10) and adopting the Gaussian normal coordinates $(b(y, t)=$ 1), we obtain the equations of motion in the following form

$$
\begin{aligned}
& \frac{3}{\kappa_{5}^{2}}\left(1-\xi \phi^{2}\right)\left(\frac{\dot{a}^{2}}{a^{2}}-\frac{n^{2} a^{\prime 2}}{a^{2}}-\frac{n^{2} a^{\prime \prime}}{a}+\frac{n^{2} k}{a^{2}}\right)+\frac{3}{\kappa_{4}^{2}}\left(\frac{\dot{a}^{2}}{a^{2}}+\frac{n^{2} k}{a^{2}}\right) \delta(y)=\frac{1}{2} \dot{\phi}^{2}+\frac{n^{2}}{2} \phi^{\prime 2}+n^{2} V(\phi) \\
& -2 n^{2} \xi\left(\phi^{\prime 2}+\phi \phi^{\prime \prime}\right)-6 n^{2} \xi\left(\frac{a^{\prime}}{a}\right) \phi \phi^{\prime}+6 \xi\left(\frac{\dot{a}}{a}\right) \phi \dot{\phi}+n^{2} \rho^{(b)} \delta(y), \\
& \gamma_{i j} \frac{a^{2}}{\kappa_{5}^{2}}\left(1-\xi \phi^{2}\right)\left[\left(\frac{a^{\prime 2}}{a^{2}}-\frac{\dot{a}^{2}}{n^{2} a^{2}}-\frac{k}{a^{2}}\right)+2\left(\frac{a^{\prime \prime}}{a}+\frac{n^{\prime} a^{\prime}}{n a}-\frac{\ddot{a}}{n^{2} a}+\frac{\dot{n} \dot{a}}{n^{3} a}+\frac{n^{\prime \prime}}{2 n}\right)\right] \\
& +\frac{\gamma_{i j}}{\kappa_{4}^{2}}\left[2\left(\frac{\dot{n} \dot{a}}{n^{3} a}-\frac{\ddot{a}}{n^{2} a}\right)-\left(\frac{\dot{a}^{2}}{n^{2} a^{2}}+\frac{k}{a^{2}}\right)\right] \delta(y)=k \gamma_{i j}\left(1-\xi \phi^{2}\right)+a^{2} \gamma_{i j}\left[\frac{1}{2 n^{2}} \dot{\phi}^{2}-\frac{1}{2} \phi^{2}-V(\phi)\right] \\
& +2 \xi \gamma_{i j}\left\{\frac{a^{2}}{n^{2}}\left[-\left(\dot{\phi}^{2}+\phi \ddot{\phi}\right)-\left(2 \frac{\dot{a}}{a}-\frac{\dot{n}}{n}\right) \phi \dot{\phi}\right]+a^{2}\left[\left(\phi^{\prime 2}+\phi \phi^{\prime \prime}\right)+\left(2 \frac{a^{\prime}}{a}+\frac{n^{\prime}}{n}\right) \phi \phi^{\prime}\right]\right\}-a^{2} \gamma_{i j} p^{(b)} \delta(y) \text {, } \\
& \frac{3}{\kappa_{5}^{2}}\left(1-\xi \phi^{2}\right)\left(\frac{n^{\prime}}{n} \frac{\dot{a}}{a}-\frac{\dot{a}^{\prime}}{a}\right)=-2 \xi\left(\phi^{\prime} \dot{\phi}+\phi \dot{\phi}^{\prime}\right)+2 \xi\left(\frac{n^{\prime}}{n}\right) \phi \dot{\phi}+\dot{\phi} \phi^{\prime} \\
& \frac{3}{\kappa_{5}^{2}}\left(\frac{a^{\prime 2}}{a^{2}}-\frac{\dot{a}^{2}}{n^{2} a^{2}}-\frac{k}{a^{2}}+\frac{n^{\prime} a^{\prime}}{n a}+\frac{\dot{n} \dot{a}}{n^{3} a}-\frac{\ddot{a}}{n^{2} a}\right)=\frac{1}{2} \phi^{\prime 2}+\frac{1}{2 n^{2}} \dot{\phi}^{2}-V(\phi)+2 \xi\left(3 \frac{a}{a^{\prime}}-\frac{n^{\prime}}{n}\right) \phi \phi^{\prime} \\
& -\frac{1}{n^{2}}\left[2 \xi\left(\dot{\phi}^{2}+\phi \ddot{\phi}\right)+2 \xi\left(3 \frac{\dot{a}}{a}-\frac{\dot{n}}{n}\right) \phi \dot{\phi}\right] \text {, }
\end{aligned}
$$

where a prime marks differentiation with respect to $y$ and a dot denotes differentiation with respect to $t$. Equations $(13)-(16)$ are $(0,0),(i, j),(0,5)$ and $(5,5)$ components of Einstein field equations respectively. Also, the scalar field evolution equation is given by

$$
\ddot{\phi}+\left(3 \frac{\dot{a}}{a}-\frac{\dot{n}}{n}\right) \dot{\phi}-n^{2}\left[\phi^{\prime \prime}+\left(\frac{n^{\prime}}{n}+3 \frac{a^{\prime}}{a}\right) \phi^{\prime}\right]+n^{2} \frac{d V}{d \phi}+\frac{n^{2}}{\kappa_{5}^{2}} \xi \phi \mathcal{R}+n^{2} \frac{\sqrt{-h}}{\sqrt{-g}} \frac{d \mathcal{L}_{b}(\phi)}{d \phi} \delta(y)=0,
$$

where the bulk Ricci scalar is defined as follows

$$
\mathcal{R}=3 \frac{k}{a^{2}}+\frac{1}{n^{2}}\left(6 \frac{\ddot{a}}{a}+6 \frac{\dot{a}^{2}}{a^{2}}-6 \frac{\dot{a} \dot{n}}{a n}\right)-6 \frac{a^{\prime \prime}}{a}-2 \frac{n^{\prime \prime}}{n}-6 \frac{a^{\prime 2}}{a^{2}}-6 \frac{a^{\prime} n^{\prime}}{a n} .
$$


As we know, in order to have a well-defined geometry, the metric is required to be continuous across the brane localized in $y=0$. However, its derivatives with respect to $y$ can be discontinuous in $y=0$. There are some terms in the Einstein tensor components that are second derivative of the metric $\left(a^{\prime \prime}, \phi^{\prime \prime}, n^{\prime \prime}\right)$. One can decompose these terms as [19]

$$
\begin{aligned}
& a^{\prime \prime}=\hat{a}^{\prime \prime}+\left[a^{\prime}\right] \delta(y), \\
& n^{\prime \prime}=\hat{n}^{\prime \prime}+\left[n^{\prime}\right] \delta(y),
\end{aligned}
$$

and

$$
a^{\prime \prime}=\hat{a}^{\prime \prime}+\left[a^{\prime}\right] \delta(y),
$$

where $\hat{A}$ shows the non-distributional part of the quantity $A$ and $[A] \equiv A\left(0^{+}\right)-A\left(0^{-}\right)$ shows the jump of this quantity across $y=0$. With this decomposition, one can equate those terms containing a Dirac delta function in the Einstein tensor with the distributional components in the stress-energy tensor. This matching leads to the following relations for the jump conditions

$$
\begin{aligned}
& \frac{\left[a^{\prime}\right]}{a_{0}}=\frac{4 \xi^{2} \phi_{0}^{2} h_{0}}{n_{0}^{2} A_{0}}+\frac{12 \xi^{2} \phi_{0}^{2} k}{a_{0}^{2} A_{0}}+ \frac{2 \kappa_{5}^{2} \xi \phi_{0}}{A_{0}} \frac{\delta \mathcal{L}(\phi)}{\delta \phi}+\frac{3 \kappa_{5}^{2} l_{0}}{n_{0}^{2} \kappa_{4}^{2} A_{0}}-\frac{\kappa_{5}^{2}}{A_{0}} \rho^{(b)}-\frac{8 \kappa_{5}^{2} \xi^{2} \phi_{0}^{2}}{\left(1-\xi \phi_{0}^{2}\right) A_{0}}\left(\rho^{(b)}+p^{(b)}\right) \\
&+\frac{16 \kappa_{5}^{2} \xi^{2} \phi_{0}^{2}}{n_{0}^{2} \kappa_{4}^{2}\left(1-\xi \phi_{0}^{2}\right) A_{0}}\left(l_{0}+m_{0} n_{0}^{2}\right), \\
& \frac{\left[\phi^{\prime}\right]}{\phi_{0}}=\frac{6 \xi h_{0}}{n_{0}^{2} \kappa_{5}^{2} A_{0}}\left(1-\xi \phi_{0}^{2}\right)+ \frac{18 \xi k}{\kappa_{5}^{2} a_{0}^{2} A_{0}}\left(1-\xi \phi_{0}^{2}\right)+\frac{3\left(1-\xi \phi_{0}^{2}\right)}{\phi_{0} A_{0}} \frac{\delta \mathcal{L}(\phi)}{\delta \phi}-\frac{24 \xi}{n_{0}^{2} \kappa_{4}^{2} A_{0}}\left(l_{0}-m_{0} n_{0}^{2}\right) \\
&-\frac{4 \xi}{A_{0}}\left(3 p^{(b)}-\rho^{(b)}\right),
\end{aligned}
$$

and

$$
\begin{gathered}
\frac{\left[n^{\prime}\right]}{n_{0}}=\frac{2 \kappa_{5}^{2} \xi \phi_{0}}{A_{0}} \frac{\delta \mathcal{L}(\phi)}{\delta \phi}+\frac{2 \kappa_{5}^{2}}{A_{0}} \rho^{(b)}+\frac{3 \kappa_{5}^{2}}{A_{0}} p+\frac{12 \xi^{2} \phi_{0}^{2} k}{a_{0}^{2} A_{0}}+\frac{4 \xi^{2} \phi_{0}^{2} h_{0}}{n_{0}^{2} A_{0}}-\frac{3 \kappa_{5}^{2} l_{0}}{n_{0}^{2} \kappa_{4}^{2} A_{0}}-\frac{6 \kappa_{5}^{2} m_{0}}{\kappa_{4}^{2} A_{0}} \\
+\frac{24 \kappa_{5}^{2} \xi^{2} \phi_{0}^{2}}{\left(1-\xi \phi_{0}^{2}\right) A_{0}}\left(\rho^{(b)}+p^{(b)}\right)-\frac{48 \kappa_{5}^{2} \xi^{2} \phi_{0}^{2}}{n_{0}^{2} \kappa_{4}^{2}\left(1-\xi \phi_{0}^{2}\right) A_{0}}\left(l_{0}+m_{0} n_{0}^{2}\right),
\end{gathered}
$$

where a prime marks differentiation with respect to $y$, a dot denotes differentiation with respect to $t$ and the subscript 0 marks quantities that are calculated at $y=0$ (on the brane). Also we have defined the following parameters

$$
\begin{aligned}
l & =\frac{\dot{a}^{2}}{a^{2}}+\frac{n^{2} k}{a^{2}}, \\
m & =\frac{\dot{n} \dot{a}}{n^{3} a}-\frac{\ddot{a}}{n^{2} a},
\end{aligned}
$$




$$
\begin{aligned}
& h=6 \frac{\ddot{a}}{a}+6 \frac{\dot{a}^{2}}{a^{2}}-6 \frac{\dot{a} \dot{n}}{a n}, \\
& A=3-3 \xi \phi^{2}+32 \xi^{2} \phi^{2} .
\end{aligned}
$$

Assuming $Z_{2}$-symmetry about the brane for simplicity, the junction conditions (22)-(24) can be used to compute $a^{\prime}, n^{\prime}$ and $\phi^{\prime}$ on two sides of the brane. It should be noticed that if we consider the case with $\xi=0$ (the minimal coupling between the scalar field and the bulk Ricci scalar), the jump conditions (22)-(24) simplify to the jump conditions achieved in Ref. $[15]$.

If we take the jump of the component $(0,5)$ of Einstein equations and use the jump conditions, we shall achieve the energy conservation equation. In our setup this equation is a complicated expression as follows

$$
\begin{gathered}
\dot{\rho}^{(b)}+3 \frac{\dot{a}_{0}}{a_{0}}\left(\rho^{(b)}+p^{(b)}\right)=-\frac{A_{0}}{3\left(1-\xi \phi^{2}\right)_{y=0}}\left\{\frac{96 \xi^{2} \phi^{2}}{A}\left(\frac{\dot{a}}{a}\right)\left(\rho^{(b)}+p^{(b)}\right)+\frac{48 \xi^{2} \phi \dot{\phi}}{A}\left(\rho^{(b)}+p^{(b)}\right)+\frac{\mathcal{X}\left(\frac{\dot{a}}{a}\right)}{3 \kappa_{4}^{2} A} h\right. \\
-\frac{2 \kappa_{5}^{2} \xi \phi \dot{\phi}}{A}\left(2 \rho^{(b)}+3 p^{(b)}\right)+\frac{\dot{\phi}}{A} \mathcal{A}\left(\rho^{(b)}-3 p^{(b)}\right)-36\left(1-\xi \phi^{2}\right) \frac{\xi^{2} \phi \dot{\phi}}{\kappa_{5}^{2} A} h+\frac{8 \xi^{3} \phi^{2} \dot{\phi}}{\kappa_{5}^{2} A}\left[3 \dot{\phi}-\kappa_{5}^{2} \phi\right] h \\
+6\left(1-\xi \phi^{2}\right)\left[\frac{\dot{\phi}}{\kappa_{5}^{2} A}\right] \mathcal{B} h+9\left(1-\xi \phi^{2}\right)\left[\frac{2 H+\frac{\dot{A}}{A}}{\kappa_{4}^{2} A}\right]\left(\frac{\dot{a}}{a}\right)^{2}-\frac{48 \xi^{2} \phi^{2}}{\kappa_{4}^{2}\left(1-\xi \phi^{2}\right) A}\left[2 \xi \phi \dot{\phi}-\kappa_{5}^{2}\right]\left(\frac{\dot{a}}{a}\right)^{2} \\
+\frac{6 \xi \phi \dot{\phi}}{\kappa_{5}^{2} A} \mathcal{D} H^{2}-\frac{18 m}{\kappa_{4}^{2} A} \mathcal{E}\left(\frac{\dot{a}}{a}\right)-\frac{48 \xi^{2} \phi^{2}}{\kappa_{4}^{2}\left(1-\xi \phi^{2}\right) A} \mathcal{K} m-\frac{12 \xi \phi \dot{\phi}}{\kappa_{4}^{2} A} \mathcal{J} m-12\left(1-\xi \phi^{2}\right) \frac{\xi^{2} \phi}{\kappa_{5}^{2} A}(\phi-\dot{\phi}) \dot{h} \\
-\frac{48 \xi^{2} \phi}{\kappa_{4}^{2} A}(\phi-\dot{\phi}) \dot{m}+3\left(1-\xi \phi^{2}\right) \frac{\mathcal{S}}{A}+\frac{2 \xi \dot{\phi} \mathcal{W}}{A} g+\frac{8 \xi^{2} \phi}{A}\left[3 \phi\left(\dot{\rho}^{(b)}+\dot{p}^{(b)}\right)+\dot{\phi}\left(3 \dot{p}-\dot{\rho}^{(b)}\right)\right] \\
\left.+\left[-3\left(1-\xi \phi^{2}\right) \rho^{(b)}-24 \xi^{2} \phi^{2}\left(\rho^{(b)}+p^{(b)}\right)\right] \frac{\dot{A}}{A}\right\}_{y=0} \equiv \Psi,
\end{gathered}
$$

where we have defined the following parameter

$$
\begin{gathered}
\mathcal{A}=8 \xi^{2} \phi+4 \xi \phi+\frac{8 \xi^{2} \phi \dot{A}}{A}-8 \xi^{2} \dot{\phi} \\
\mathcal{B}=-2 \xi^{2} \dot{\phi}+\frac{2 \xi^{2} \phi \dot{A}}{A}+\frac{2 \xi^{2} \phi^{2} \dot{A}}{\dot{\phi} A}+\xi \phi \\
\mathcal{D}=-\frac{32 \xi \phi}{\dot{\phi}} H-8 \xi+\frac{8 \xi \phi \dot{A}}{\dot{\phi} A}-4+\frac{8 \xi \dot{\phi}}{\phi}-\frac{8 \dot{A}}{A}+\kappa_{5}^{2}, \\
\mathcal{E}=1-\xi \phi^{2}+\frac{32}{3} \xi^{2} \phi^{2}, \\
\mathcal{K}=2 \xi \phi \dot{\phi}+\kappa_{5}^{2},
\end{gathered}
$$




$$
\begin{gathered}
\mathcal{J}=12 \xi-4 \xi \frac{\dot{\phi}}{\dot{\phi} A}-2+4 \xi \frac{\dot{\phi}}{\phi}-4 \xi \frac{\dot{A}}{A}-\kappa_{5}^{2}, \\
\mathcal{X}=-9+9 \xi \phi^{2}-48 \xi^{2} \phi^{2}+48 \xi^{2} \phi \dot{\phi}, \\
\mathcal{S}=2 \xi \phi \dot{g}+4 \xi \dot{\phi} g-2 \xi \phi g \frac{\dot{A}}{A}-\dot{\phi} g+2 \xi \dot{\phi} \dot{g}, \\
\mathcal{W}=-6 \xi \phi-3 \frac{\dot{A}}{A}+2 \kappa_{5}^{2} \phi^{2},
\end{gathered}
$$

and

$$
g=\frac{\delta \mathcal{L}_{b}(\phi)}{\delta \phi} .
$$

Also the subscript 0 indicates the parameter on the brane. The right hand side of the conservation equation is non-zero and this means that the energy on the brane is not conserved, i.e. there may be leakage of energy-momentum from the brane or suction onto the brane. In this respect, for negative values of $\Psi$, energy-momentum leaks off the brane and for positive $\Psi$ energy-momentum flows from the bulk onto the brane.

\section{DGP braneworld cosmology with a non-minimally coupled bulk scalar field}

Now we follow Ref. [20] to obtain a special class of solutions for a DGP braneworld cosmology with a non-minimally coupled bulk scalar field. In this regard, we introduce the quantity $\mathcal{F}$ as a function of $t$ and $y$ as follows

$$
\mathcal{F}(t, y)=\left(\frac{a^{\prime}}{a b}\right)^{2}-\left(\frac{\dot{a}}{a n}\right)^{2}
$$

So, we can rewrite the components $(0,0)$ and $(5,5)$ of the Einstein's field equations in the bulk as follows

$$
\begin{aligned}
& G_{0}^{0}-\frac{\dot{a}}{a^{\prime}} G_{5}^{0}=\left(1-\xi \phi^{2}\right) \frac{3}{2 a^{3} a^{\prime}} \partial_{y}\left(a^{4} \mathcal{F}\right) \\
& G_{5}^{5}-\frac{a^{\prime}}{\dot{a}} G_{0}^{5}=\left(1-\xi \phi^{2}\right) \frac{3}{2 a^{3} \dot{a}} \partial_{t}\left(a^{4} \mathcal{F}\right)
\end{aligned}
$$

where $G_{A B}$ is defined as

$$
G_{A B}=\left(1-\xi \phi^{2}\right)\left(\mathcal{R}_{A B}-\frac{1}{2} g_{A B} \mathcal{R}\right)
$$

In the presence of the bulk scalar field, the left hand sides of these two equations are not the same. But for special class of solutions with $\phi=\phi(a)$, they are equivalent and in this case $\mathcal{F}=\mathcal{F}(a)$. In this situation, both (41) and (42) reduce to

$$
6\left(1-\xi \phi^{2}\right) \mathcal{F}+\frac{3}{2}\left(1-\xi \phi^{2}\right)\left(a \frac{d \mathcal{F}}{d a}\right)+\frac{\kappa_{5}^{2}}{2} \mathcal{F}\left(a \frac{d \phi}{d a}\right)^{2}-2 \kappa_{5}^{2} \xi \mathcal{F}\left(a \frac{d \phi}{d a}\right)^{2}-2 \kappa_{5}^{2} \xi \mathcal{F} \phi a^{2}\left(\frac{d^{2} \phi}{d a^{2}}\right)
$$




$$
-6 \kappa_{5}^{2} \xi \phi \mathcal{F}\left(a \frac{d \phi}{d a}\right)-\kappa_{5}^{2} \xi \phi\left(a \frac{d \mathcal{F}}{d a}\right)\left(a \frac{d \phi}{d a}\right)-\frac{2 \kappa_{5}^{2} \xi \phi}{a} \mathcal{F}+\kappa_{5}^{2} V(\phi)=0 .
$$

We choose a Gaussian normal coordinate system so that $b^{2}(y, t)=1$. Also we assume that $t$ as a proper cosmological time on the brane has scaled so that $n_{0}=1$. By adopting a $Z_{2}$ symmetry across the brane, equations (13) and (16) yield the following generalization of the Friedmann equation for cosmological dynamics on the DGP brane

$$
\begin{gathered}
3 \kappa_{5}^{2}\left(\xi \phi_{0}^{2}-1\right) H^{2}+24 \xi^{2} \phi_{0}^{2} \kappa_{4}^{2}\left(\xi \phi_{0}^{2}-1\right) H^{2}+8 \xi^{2} \phi_{0}^{2} \kappa_{5}^{2} \kappa_{4}^{2}\left(\rho^{(b)}+p^{(b)}\right)-16 \xi^{2} \phi_{0}^{2} \kappa_{5}^{2}\left(m_{0}+H^{2}\right) \\
+\rho^{(b)} \kappa_{5}^{2} \kappa_{4}^{2}\left(1-\xi \phi_{0}^{2}\right)+2 \xi \phi_{0} \kappa_{4}^{2} \kappa_{5}^{2}\left(\xi \phi_{0}^{2}-1\right) g_{0}+24 \xi^{2} \phi_{0}^{2} \kappa_{4}^{2}\left(1-\xi \phi_{0}^{2}\right) m_{0}= \pm 2 \kappa_{4}^{2}\left(1-\xi \phi_{0}^{2}\right) A \sqrt{H^{2}-\mathcal{F}_{0}}
\end{gathered}
$$

where $H=\left(\frac{\dot{a}}{a}\right)_{y=0}$ and \pm refers to two branches of the DGP model. We have solved this equation for $H^{2}$ and the result is presented in Appendix A. If we consider minimal coupling of the scalar field and 5D Ricci scalar $(\xi=0)$, we reach the following Friedmann equation on the brane which was obtained in [15]

$$
H^{2}=\frac{1}{3} \kappa_{4}^{2} \rho^{(b)}+\frac{2 \kappa_{4}^{4}}{\kappa_{5}^{4}} \pm \frac{2 \kappa_{4}^{2}}{\kappa_{5}^{2}} \sqrt{\frac{\kappa_{4}^{4}}{\kappa_{5}^{4}}+\frac{1}{3} \kappa_{4}^{2} \rho^{(b)}+\mathcal{F}_{0}} .
$$

We note that from now on we consider only the normal, ghost-free branch of the Friedmann equation. Since we consider $\phi=\phi(a)$, the scalar field equation (7) reduces to

$$
\nabla^{2} \phi=a^{2}\left(\frac{d^{2} \phi}{d a^{2}}+\frac{1}{a} \frac{d \phi}{d a}\right) \mathcal{F}+\left[\frac{a\left(G_{0}^{0}+G_{5}^{5}\right)}{3\left(1-\xi \phi^{2}\right)}\right] \frac{d \phi}{d a}=-\frac{1}{\kappa_{5}^{2}} \xi \phi \mathcal{R}+\frac{d V}{d \phi}-\delta(y) \frac{\sqrt{-h}}{\sqrt{-g}} \frac{\delta \mathcal{L}_{b}(\phi)}{\delta \phi} .
$$

Now, by substituting equations $G_{A B}=\kappa_{5}^{2} T_{A B}$ and (44) into (47), we find

$$
\begin{gathered}
\frac{2 a}{3\left(1-\xi \phi^{2}\right)}\left\{6\left(1-\xi \phi^{2}\right) \mathcal{F}+\frac{3}{2}\left(1-\xi \phi^{2}\right)\left(a \frac{d \mathcal{F}}{d a}\right)+\frac{\kappa_{5}^{2}}{2} \mathcal{F}\left(a \frac{d \phi}{d a}\right)^{2}-2 \kappa_{5}^{2} \xi \mathcal{F} \phi a^{2}\left(\frac{d^{2} \phi}{d a^{2}}\right)-\frac{2 \kappa_{5}^{2} \xi \phi}{a} \mathcal{F}\right. \\
\left.-2 \kappa_{5}^{2} \xi \mathcal{F}\left(a \frac{d \phi}{d a}\right)^{2}-6 \kappa_{5}^{2} \xi \phi \mathcal{F}\left(a \frac{d \phi}{d a}\right)-\kappa_{5}^{2} \xi \phi\left(a \frac{d \mathcal{F}}{d a}\right)\left(a \frac{d \phi}{d a}\right)+2 \kappa_{5}^{2} \xi \phi\left(a^{\prime \prime}+\frac{n^{\prime} a^{\prime}}{a}-\frac{\ddot{a}}{n^{2}}\right)\left(\frac{d \phi}{d a}\right)\right\} \frac{d \phi}{d a} \\
+\mathcal{F}\left(a \frac{d}{d a}\right)^{2} \phi+\frac{1}{\kappa_{5}^{2}} \xi \phi \mathcal{R}-\frac{d V}{d \phi}+\delta(y) \frac{\sqrt{-h}}{\sqrt{-g}} \frac{\delta \mathcal{L}_{b}(\phi)}{\delta \phi}=0
\end{gathered}
$$

Thus the original partial differential field equations have been reduced to an ordinary differential equation.

\section{Supergravity-style solutions}

In this section we are going to generate some special solutions of the field equations. In this respect, one way is to introduce a special supergravity-style potential, $V(\phi)$, as follows [21]

$$
V(\phi)=\frac{1}{8}\left(\frac{d W}{d \phi}\right)^{2}-\frac{\kappa_{5}^{2}}{6} W^{2} .
$$


Assuming $k=0$, the field equations (44) and (48) are satisfied if

$$
\begin{gathered}
\mathcal{F}=\frac{\kappa_{5}^{4}}{36} W^{2} \\
\frac{d^{2} \phi}{d a^{2}}=-3 \frac{\phi}{\kappa_{5}^{2} a^{2}}+\frac{3}{2} \frac{\left(\frac{d W}{d \phi}\right)\left(\frac{d \phi}{d a}\right)}{\kappa_{5}^{2} \xi W \phi a}-\frac{3}{2} \frac{\phi\left(\frac{d W}{d \phi}\right)\left(\frac{d \phi}{d a}\right)}{\kappa_{5}^{2} W a}+\frac{1}{4} \frac{\left(\frac{d \phi}{d a}\right)^{2}}{\xi \phi}-\frac{\left(\frac{d \phi}{d a}\right)^{2}}{\phi} \\
-3 \frac{\left(\frac{d \phi}{d a}\right)}{a}-\frac{\left(\frac{d \phi}{d a}\right)^{2}\left(\frac{d W}{d \phi}\right)}{W}-a^{-3}+\frac{9}{4} \frac{\left(\frac{d W}{d \phi}\right)^{2}}{\xi \kappa_{5}^{2} W^{2} \phi a^{2}} \equiv \mathcal{Q}
\end{gathered}
$$

Equation (51) is a generalization of the result obtained in Refs. [15,21]. This equation helps us to find the necessary condition for the consistency of the jump conditions. From equation (51) we deduce the following equation

$$
\frac{d \phi}{d a}=\Upsilon
$$

where $\Upsilon=\int \mathcal{Q} d a$ and $\mathcal{Q}$ is defined in Eq. (51). By using of Eqs. (22), (23) and (52) we find that the consistency of jump conditions for a $Z_{2}$-symmetric DGP brane is guaranteed if

$$
\begin{aligned}
& \frac{\delta \mathcal{L}_{b}(\phi)}{\delta \phi}=\frac{A_{0}}{3\left(1-\xi \phi_{0}^{2}\right)-2 \Upsilon_{0} \kappa_{5}^{2} \xi \phi_{0} a_{0}}\left\{\frac{4 \xi \phi_{0}}{A_{0}}\left(3 p^{(b)}-\rho^{(b)}\right)-\frac{6 \xi h_{0} \phi_{0}}{n_{0}^{2} \kappa_{5}^{2} A_{0}}\left(1-\xi \phi_{0}^{2}\right)+\frac{24 \xi \phi_{0}}{n_{0}^{2} \kappa_{4}^{2} A_{0}}\left(l_{0}-m_{0} n_{0}^{2}\right)\right. \\
& \left.+\Upsilon_{0} a_{0}\left[\frac{4 \xi^{2} \phi_{0}^{2} h_{0}}{n_{0}^{2} A_{0}}+\frac{3 \kappa_{5}^{2} l_{0}}{n_{0}^{2} \kappa_{4}^{2} A_{0}}-\frac{\kappa_{5}^{2}}{A_{0}} \rho^{(b)}-\frac{8 \kappa_{5}^{2} \xi^{2} \phi_{0}^{2}}{\left(1-\xi \phi_{0}^{2}\right) A_{0}}\left(\rho^{(b)}+p^{(b)}\right)+\frac{16 \kappa_{5}^{2} \xi^{2} \phi_{0}^{2}\left(l_{0}+m_{0} n_{0}^{2}\right)}{n_{0}^{2} \kappa_{4}^{2}\left(1-\xi \phi_{0}^{2}\right) A_{0}}\right]\right\} .
\end{aligned}
$$

It should be noticed that if there is $\phi$ dependent couplings in the standard model Lagrangian, $\mathcal{L}_{b}$ satisfies the above condition. Equation (53) in the minimal case with $\xi=0$ simplifies to (see [15])

$$
\frac{\delta \mathcal{L}_{b}(\phi)}{\delta \phi}=\left(-\frac{\kappa_{5}^{2}}{6} \rho^{(b)}+\frac{\kappa_{5}^{2}}{2 \kappa_{4}^{2}} \frac{\dot{a}_{0}^{2}}{a_{0}^{2}}\right)\left(-\frac{6}{\kappa_{5}^{2} W} \frac{d W}{d \phi}\right)_{0} .
$$

\subsection{Exponential Superpotentials}

In this section, we consider the following exponential form of the superpotential which is motivated by string/M-theory [21]

$$
W=c\left[\frac{e^{-\alpha_{1} \phi}}{\alpha_{1}}+s \frac{e^{\alpha_{2} \phi}}{\alpha_{2}}\right],
$$

where $s= \pm 1$ and $\alpha_{1} \geq\left|\alpha_{2}\right|$. For $\alpha_{2}=0$, we use the following form of the superpotential

$$
W=c\left[\frac{e^{-\alpha_{1} \phi}}{\alpha_{1}}+\frac{s}{\kappa_{5}}\right] .
$$


The corresponding potentials obtained from (49) are

$$
V=\frac{c^{2}}{8}\left[\left(1-\frac{4 \kappa_{5}^{2}}{3 \alpha_{1}^{2}}\right) e^{-2 \alpha_{1} \phi}+\left(1-\frac{4 \kappa_{5}^{2}}{3 \alpha_{2}^{2}}\right) e^{2 \alpha_{2} \phi}-2 s\left(1+\frac{4 \kappa_{5}^{2}}{3 \alpha_{1} \alpha_{2}}\right) e^{\left(\alpha_{2}-\alpha_{1}\right) \phi}\right] .
$$

and (for $\left.\alpha_{2}=0\right)$

$$
V=\frac{c^{2}}{8}\left[\left(1-\frac{4 \kappa_{5}^{2}}{3 \alpha_{1}^{2}}\right) e^{-2 \alpha_{1} \phi}-2 s \frac{4 \kappa_{5}}{3 \alpha_{1}} e^{-\alpha_{1} \phi}-\frac{4}{3}\right] .
$$

For $V$ bounded from below, only some values of the parameters are allowed [21].

By using these exponential superpotentials and also equations (49) and (51) one can deduce the evolution of the scalar field with respect to scale factor and therefore the cosmology of the model is obtained. Equation (51) is a complicated nonlinear second order differential equation with no analytical solutions. So, we have solved this equation numerically the results of which are shown in forthcoming figures. Depending on the value of $s$ and $\alpha_{2}$ and also the sign of $\alpha_{2}$, there will be a variety of cosmological evolution on the brane with several interesting implications. To be more specific, in which follows we discuss each of these choices of $s$ and $\alpha_{2}$ separately. We note that the solutions which start at $a=0$ are interesting because these solutions provide a big bang style cosmology.

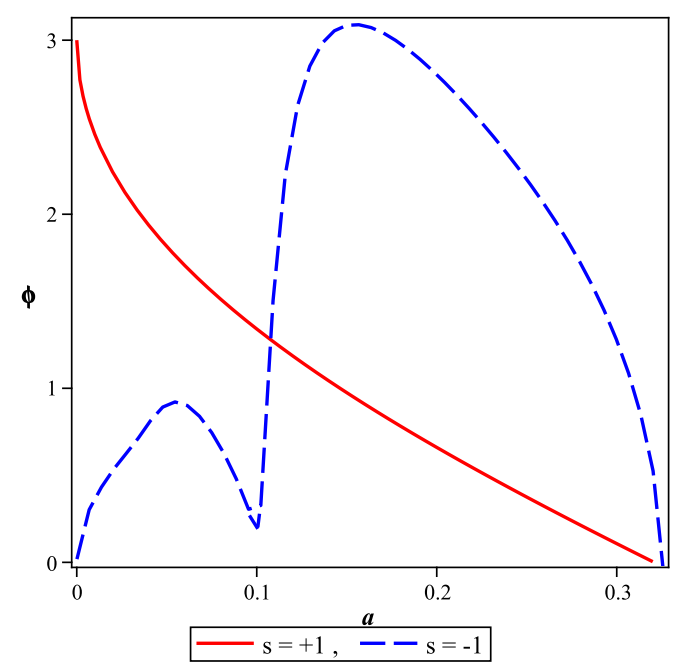

Figure 1: Evolution of the scalar field with respect to the scale factor for the case with $\alpha_{2}=+1$ and $\alpha_{1}=+2$. In the case with $s=+1$ (the red-solid line), the scalar field decreases with scale factor until in some value of $a$ it tends to zero. In the case with $s=-1$ (blue-dashed line), as scale factor increases, the scalar field increases firstly and then decreases. By more increment of the scale factor, $\phi$ increases again and then decreases towards zero. 


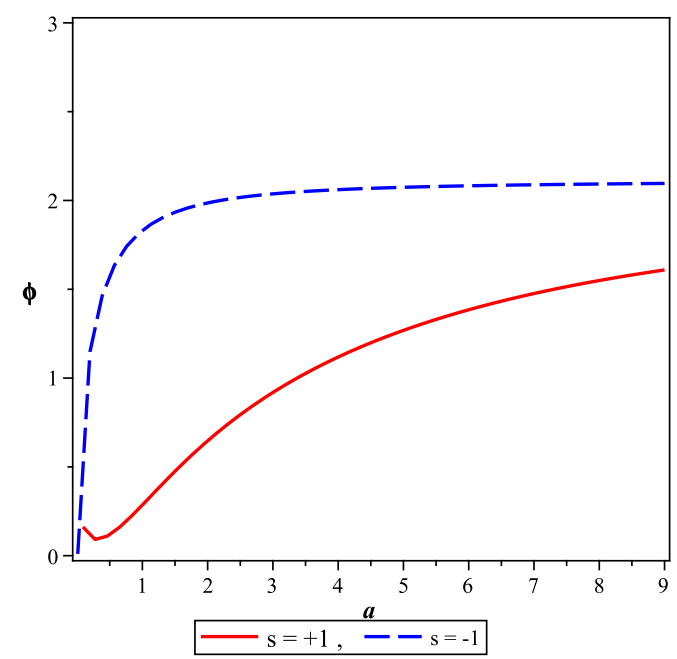

Figure 2: Evolution of the scalar field versus the scale factor for $\alpha_{2}=-1$ and $\alpha_{1}=+2$. In the case with $s=+1$ (the red-solid line), the scalar field decreases firstly with scale factor towards a minimum and then increases. In the case with $s=-1$ (the blue-dashed line), as scale factor increases, the scalar field increases too.

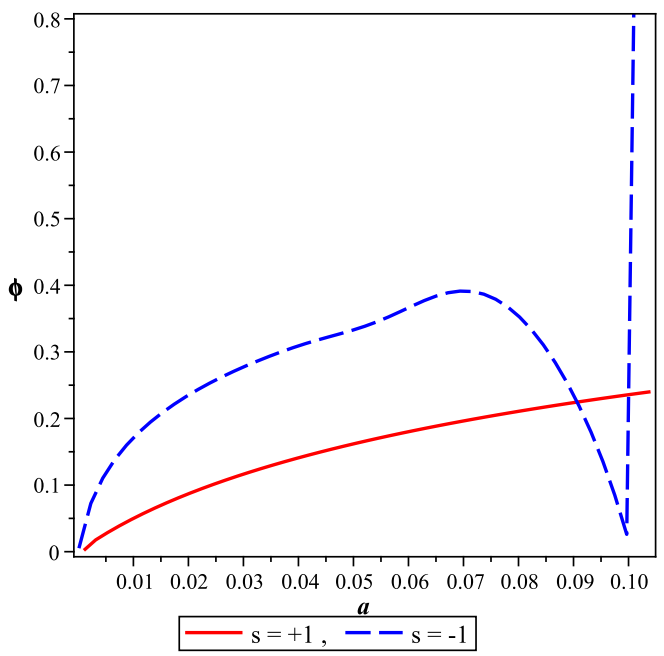

Figure 3: Evolution of the scalar field versus the scale factor for $\alpha_{2}=0$ and $\alpha_{1}=+2$. With $s=+1$ (the red-solid line), the scalar field increases with scale factor. With $s=-1$ (the blue-dashed line), as scale factor increases, the scalar field decreases firstly and then decreases towards a minimum. After that it increases rapidly by more increment of the scale factor. 
- $\alpha_{2}>0$ :

First, we choose $\alpha_{1}=+2$ and $\alpha_{2}=+1$. In this case we obtain the evolution of the scalar field with respect to the scale factor for both $s=+1$ and $s=-1$. As figure 1 shows, for $s=+1$ the scalar field decreases by increment of the scale factor and tends to zero at some values of the scale factor, but for $s=-1$ the behavior of the scalar field is different. For $s=-1$, at first, the scalar field increases by the scale factor until a maximum and then begins reduction. After that, it increases again and then experiences another reduction until reaches zero at some values of the scale factor. Since we expect that the scalar field to reduce by scale factor and tend to zero finally, so it seems that these choice of values for $\alpha_{1}, \alpha_{2}$ and $s$ are reliable. It should be noted that in plotting this figure (and also forthcoming figures) we have set $\xi=\frac{1}{6}, \kappa_{5}=1$, $\kappa_{4}=1$ and $c=1$.

\section{- $\alpha_{2}<0$ :}

Next, we choose $\alpha_{1}=+2$ and $\alpha_{2}=-1$. In this case we obtain the evolution of the scalar versus the scale factor for both $s=+1$ and $s=-1$, the results of which are shown in figure 2. For $s=+1$, the scalar field decreases firstly while the scale factor increases and then increases until scale factor tends to infinity. For $s=-1$, the scalar field starts at $a=0$ and increases as the scale factor tends to infinity. So, it seems that these choice of values for $\alpha_{1}, \alpha_{2}$ and $s$ cannot lead to a cosmologically viable result.

\section{- $\alpha_{2}=0$ :}

Finally, we choose $\alpha_{1}=+2$ and $\alpha_{2}=0$, so we use equation (56) for superpotential. Figure 3 shows the behavior of the scalar field versus the scale factor for $s=+1$ and $s=-1$. For $s=+1$ the scalar field starts from zero at $a=0$ and increases by scale factor. But for $s=-1$, the scalar field starts at $a=0$ and increases by scale factor towards a maximum, then reduces by more increment of the scale factor and finally experiences a rapid increment again. So, these choice of values for $\alpha_{1}, \alpha_{2}$ and $s$ cannot lead one to a cosmologically viable result.

We note that in the minimal case, just for $\alpha_{2}>0$ and $s=+1$, the scalar field reduces by increment of the scale factor and tends to zero in some value of $a$ (see [15]).

As we have said earlier, the presence of a bulk scalar field (specially as in our setup with a non-minimally coupled bulk scalar field) causes the non-conservation of the energymomentum tensor on the brane (that is, the bulk-brane energy-momentum exchange). Since the right hand side of Eq. (29) shows this non-conservation, we have performed some numerical analysis on this equation to explore some of its physical implications. We obtain the evolution of $\Psi$ with respect to the cosmic time on the brane in order to clarify if by expansion of the universe, there is leakage of energy from the brane into the bulk or energy suction occurs onto the brane from the bulk. Note that since the case with $\alpha_{2}>0$ and $s= \pm 1$ for 


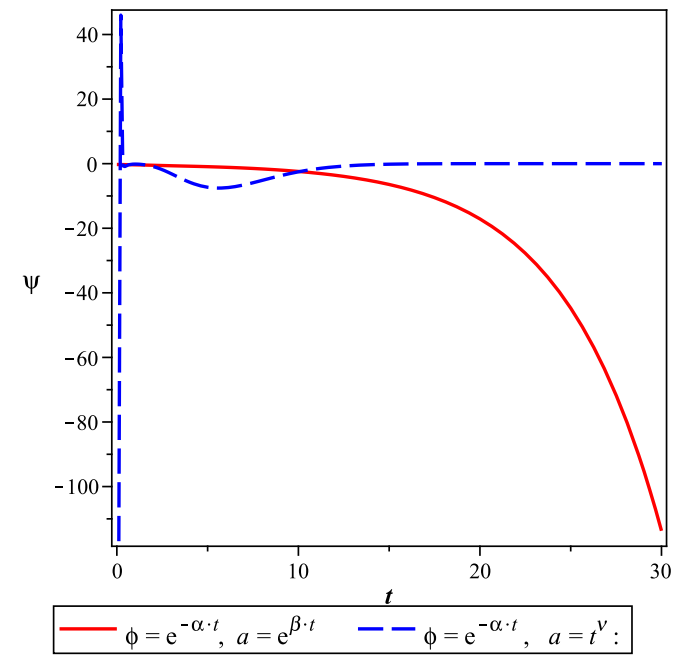

Figure 4: Evolution of $\Psi$ versus the scalar field (corresponding to $s=+1$ ). The behavior of this parameter clarifies the status of the energy conservation. In the case with $a=e^{\beta t}$ (the red-solid line), $\Psi$ is always negative. This states that as the universe expands, the energy leaks off the brane into the bulk. In the case with $a=t^{\nu}$ (the blue-dashed line), $\Psi$ is positive in some time intervals. This means that in these intervals, the energy is sucked onto the brane by expansion of the universe.

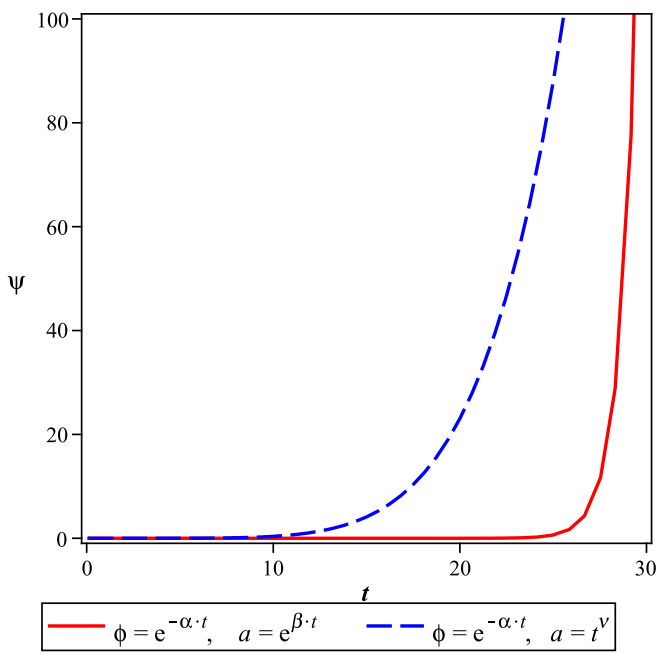

Figure 5: Evolution of $\Psi$ versus the scalar field (corresponding to $s=-1$ ). In both cases with $a=e^{\beta t}$ (the red-solid line) and $a=t^{\nu}$ (the blue-dashed line), $\Psi$ is always positive. This states that as the universe expands, suction of energy onto the brane occurs. 
superpotential are more favorable in cosmological grounds, we perform our analysis just for the mentioned choice of parameters. Also we consider one ansatz for the scalar field and two for scale factor as follows

$$
\phi=\phi_{0} e^{-\alpha t}, \quad a=a_{0} e^{\beta t}, \quad a=a_{0} t^{\nu}
$$

where $\alpha, \beta$ and $\mu$ are positive constants. We have considered the simplest generalization of the brane energy density as

$$
\rho^{(b)}=W_{0} \rho
$$

where $\rho$ is proportional to the energy density of the ordinary matter on the brane. This generalization has its origin in the fact that matter Lagrangian on the brane, that is $\mathcal{L}_{b}(\phi)$, depends on the bulk scalar field, $\phi$. We have assumed that the ordinary matter on the brane is dust with $\omega=0$ and $\rho=\rho_{0} a^{-3}$. With these assumptions, we have performed our numerical analysis and the results are as shown in figures 4 and 5 .

- $s=+1$ :

In the case with $s=+1$ and for $a=a_{0} e^{\beta t}, \Psi$ is negative always. So, as time passes and the universe expands, the energy leaks off the brane. For $a=a_{0} t^{\nu}$, in some time interval $\Psi$ is negative while it is positive in some other time intervals. So, in some time interval we have leakage of energy from the brane into the bulk and in some other time interval energy is sucked onto the brane from the bulk.

- $s=-1$ :

In the case with $s=-1$ and for both $a=a_{0} e^{\beta t}$ and $a=a_{0} t^{\nu}$, in all times $\Psi$ is positive and this means that, for these choices of parameters, as the universe expands there is the energy suction onto the brane.

So, it seems that the case with positive $\alpha_{2}$ and $s=+1$ with exponentially evolving scalar field and scale factor leads to a viable cosmology.

\section{Late Time Cosmology}

In this section we study the effect of a non-minimally coupled bulk scalar field on the late time behavior in the normal branch of a DGP-inspired braneworld model. In this regard, we firstly rewrite the Friedmann equation (45) (see also Appendix A) in some simpler form. Since we want to study the late time behavior, so we deal with the small scalar field regime. On the other hand, the coupling constant, $\xi$, is small $\left(\xi=\frac{1}{6}\right)$ so it is sufficient to preserve the terms up to the order of $\xi^{2}$. Therefore the Friedmann equation simplifies to the following form

$$
H^{2}=\frac{1}{\left(-3 \kappa_{5}^{2}-16 \xi^{2} \phi_{0}^{2} \kappa_{5}^{2}+3 \xi \phi_{0}^{2} \kappa_{5}^{2}-24 \xi^{2} \phi_{0}^{2} \kappa_{4}^{2}\right)^{2}}\left\{384 \xi^{2} \phi^{2} \kappa_{4}^{4}-48 \xi^{2} \phi^{2} \kappa_{5}^{4} m+108 \kappa_{4}^{4} \xi^{2} \phi^{4}\right.
$$




$$
\begin{gathered}
+3 \rho^{(b)} \kappa_{5}^{4} \kappa_{4}^{2}-72 \kappa_{4}^{4} \xi \phi^{2}+18 \kappa_{4}^{4}-6 \xi \phi^{2} \kappa_{5}^{4} \rho^{(b)} \kappa_{4}^{2}+12 \xi^{2} \phi^{3} \kappa_{4}^{2} g \kappa_{5}^{4}+40 \xi^{2} \phi^{2} \rho^{(b)} \kappa_{5}^{4} \kappa_{4}^{2}-6 \xi \phi \kappa_{4}^{2} g \kappa_{5}^{4} \\
+72 \xi^{2} \phi^{2} \kappa_{4}^{2} m \kappa_{5}^{2}+3 \xi^{2} \phi^{4} \kappa_{5}^{4} \rho^{(b)} \kappa_{4}^{2}+24 \xi^{2} \phi^{2} \kappa_{4}^{4} \rho^{(b)} \kappa_{5}^{2}+24 \xi^{2} \phi^{2} p \kappa_{5}^{4} \kappa_{4}^{2} \\
-6 \kappa_{4}^{2}\left[24 \xi^{2} \phi^{2} \kappa_{4}^{4} \rho \kappa_{5}^{2}-72 \kappa_{4}^{4} \xi \phi^{2}+252 \kappa_{4}^{4} \xi^{2} \phi^{4}+9 \kappa_{4}^{4}+72 \xi^{2} \phi^{2} \kappa_{4}^{2} m \kappa_{5}^{2}-18 \xi \phi^{2} \kappa_{5}^{4} \rho \kappa_{4}^{2}\right. \\
-6 \xi \phi \kappa_{4}^{2} g \kappa_{5}^{4}+24 \xi^{2} \phi^{2} p \kappa_{5}^{4} \kappa_{4}^{2}+36 \xi^{2} \phi^{3} \kappa_{4}^{2} g \kappa_{5}^{4}+104 \xi^{2} \phi^{2} \rho \kappa_{5}^{4} \kappa_{4}^{2}+45 \xi^{2} \phi^{4} \kappa_{5}^{4} \rho \kappa_{4}^{2}+144 \xi^{2} \phi^{2} \kappa_{4}^{2} \kappa_{5}^{2} \mathcal{F} \\
\left.\left.+3 \rho \kappa_{5}^{4} \kappa_{4}^{2}+9 \kappa_{5}^{4} \mathcal{F}-48 \xi^{2} \phi^{2} \kappa_{5}^{4} m+288 \kappa_{5}^{4} \mathcal{F} \xi^{2} \phi^{2}+135 \kappa_{5}^{4} \mathcal{F} \xi^{2} \phi^{4}-54 \kappa_{5}^{4} \mathcal{F} \xi \phi^{2}\right]^{\frac{1}{2}}\right\}_{y=0}
\end{gathered}
$$

Now we rewrite the Friedmann equation (61) in the form of an effective Friedmann equation as follows

$$
H^{2}=\frac{\kappa_{4}^{2}}{3}\left(\rho_{m}+\rho_{e f f}\right)
$$

where $\rho_{m}$ is the standard matter energy density and $\rho_{e f f}$ is the energy density corresponding to the dark energy sector of the model. One of the properties of phantom-like behavior is that the effective energy density of the model grows with time (in other words, it grows by reduction of the red-shift parameter, $z$ ). We avoid to write the equation of the effective energy density since it has a simple relation with $H^{2}$ and there is no need to write it again. We just show the behavior of the $\rho_{\text {eff }}$ with respect to the red-shift in figure 6 . It is obvious that for both $a=a_{0} e^{\beta t}$ and $a=a_{0} t^{\nu}$, the effective energy density grows by cosmic expansion (decreasing $z$ ). It should be noted that in plotting this figure (and also, other figures), we have used the relation $1+z=\frac{a_{0}}{a}$.

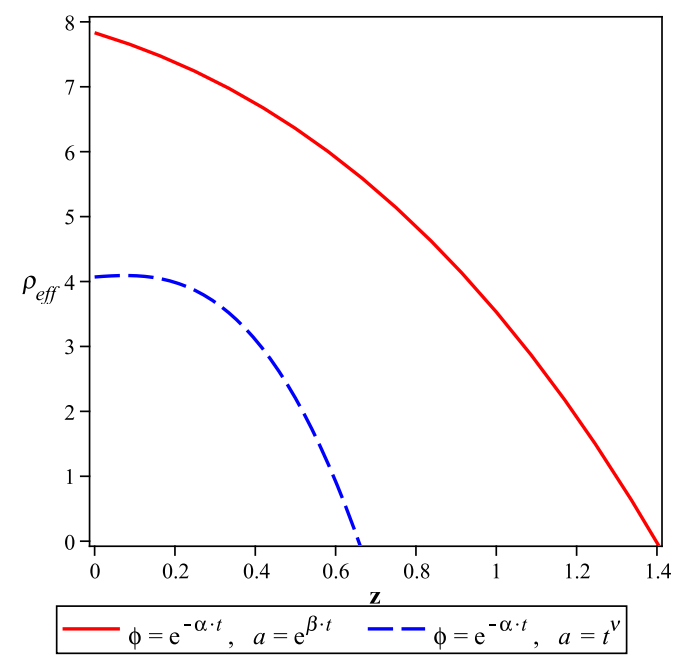

Figure 6: Variation of the effective dark energy density versus the redshift. The red-solid line is for the case with $\phi=e^{-\alpha t}$ and $a=e^{\beta t}$. The blue-dashed line is for the case with $\phi=e^{-\alpha t}$ and $a=t^{\nu t}$. In both cases, the effective energy density increases when red-shift decreases. 


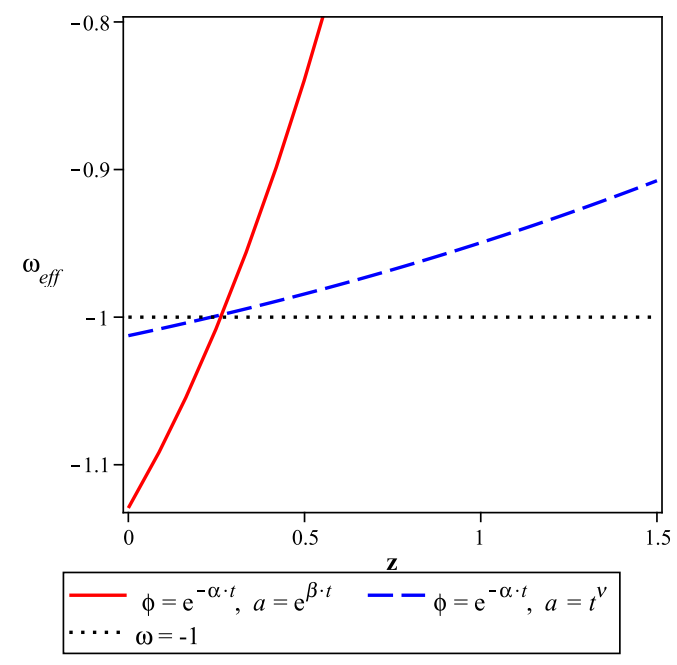

Figure 7: Variation of $\omega_{\text {eff }}$ versus the redshift. The red-solid line is for the case with $\phi=e^{-\alpha t}$ and $a=e^{\beta t}$. The blue-dashed line is for the case with $\phi=e^{-\alpha t}$ and $a=t^{\nu t}$. In both cases, $\omega_{\text {eff }}$ crosses $\omega=-1$ line in $z \simeq 0.26$.

Time evolution of the equation of state parameter gives us a suitable background to understand the nature of dark energy. The $\Lambda$ CDM model as a candidate for dark energy has good agreement with the recent observational data [22]. However, recent observational data show also that the dark energy component has an equation of state parameter $\omega<-1$ at the present epoch, while $\omega>-1$ in the past. One way to explain these observations is to consider a dynamical dark energy component with transient equation of state parameter [3]. The dynamical dark energy component with $\omega<-1$ has a phantom nature. A cosmological model based on the phantom fields suffers from instabilities; a phantom universe ends up with a Big Rip singularity. It is due to the fact that energy density for these fields is a growing function of the scale factor in an expanding FRW universe. It has been shown that the normal branch of the DGP scenario has the potential to explain a phantom-like behavior on the brane without introducing any phantom fields neither in the bulk nor on the brane [11]. In order to considering the evolution of the equation of state parameter in our setup, we use the effective conservation equation as

$$
\dot{\rho}_{e f f}+3 H \rho_{e f f}\left(1+\omega_{e f f}\right)=0 .
$$

Note that, equation (63) is an effective conservation equation and the effect of non-minimally coupled scalar field is hidden in the definition of $\rho_{\text {eff }}$. By using equations (62) and (63), we have derived the effective equation of state parameter as has been shown in Appendix B. The behavior of $\omega_{\text {eff }}$ with respect to the red-shift parameter is shown in figure 7 . This figure shows that in this scenario, with both $a=a_{0} e^{\beta t}$ and $a=a_{0} t^{\nu}$, the universe enters the phantom phase in the near past and currently it is in the phantom phase. The transition from quintessence to the phantom phase has occurred at $z=0.26$. So, this model experiences a smooth crossing of the phantom divide, $\omega_{\text {eff }}=-1$, line. 


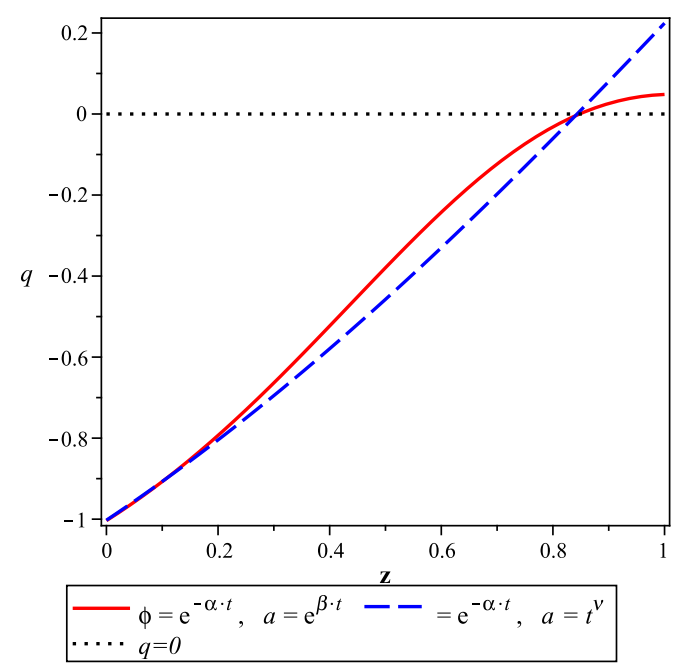

Figure 8: Variation of the deceleration parameter versus the redshift. The red-solid line is for the case with $\phi=e^{-\alpha t}$ and $a=e^{\beta t}$. The blue-dashed line is for the case with $\phi=e^{-\alpha t}$ and $a=t^{\nu t}$. Figure shows that the universe, with both types of scale factors, has entered the accelerating phase at $z \simeq 0.84$

Another important parameter in cosmological evolution is the deceleration parameter which is defined as

$$
q=-\left[\frac{\dot{H}}{H^{2}}+1\right] .
$$

A positive value of this parameter corresponds to $\ddot{a}<0$, and this means that the universe expansion is decelerating. A negative value of $q$ corresponding to $\ddot{a}>0$, means that the universe expansion is positively accelerated. The calculation of the deceleration parameter in our setup is presented in Appendix $\mathbf{C}$ and the behavior of $q$ versus $z$ is shown in figure 9. This figure shows that the deceleration parameter becomes negative at $z \simeq 0.84$ and this means that the universe has entered into an accelerating phase in the past at $z \simeq 0.84$. So, we can say that a warped DGP model, in the presence of a non-minimally coupled bulk scalar field, has the phantom like behavior and can explain the late time cosmic acceleration of the universe in an observationally viable manner.

\section{Summary}

In this paper, we have studied the cosmological dynamics of a bulk scalar field which is non-minimally coupled with 5D intrinsic curvature in the DGP setup. We have derived the Bulk-brane Einstein's equations and the scalar field's equation of motion in this scenario. Then, by decomposing those components of the Einstein tensor that are second derivative of the metric and matching the resulting terms which contain Dirac delta function with the distributional parts of the stress-energy tensor, we have derived the jump conditions in this 
setup. We have found that the presence of non-minimally coupled bulk scalar field, changes the original jump condition of the DGP model and even changes the jump conditions in the warped DGP model with a minimally coupled bulk scalar field. By using the resulting jump conditions we have derived the energy conservation equation in our setup. The non-vanishing right hand side of this equation shows the bulk brane energy exchange in the presence of the bulk scalar field (minimally or non-minimally coupled with curvature). To obtain a special class of solutions for a DGP braneworld cosmology with a bulk scalar field, by introducing the quantity $\mathcal{F}$ as a function of $t$ and $y$, we have found the Friedmann equation of the model and also we reduced the original partial differential field equations to an ordinary differential equation. We have used the superpotential method in order to generate some solutions of the field equations of the model. By choosing an exponential superpotential we have performed some numerical analysis on the model parameters space. Since the self-accelerating DGP branch has ghost instabilities, we restricted our study to the normal DGP branch of this DGP-inspired model. We have found that just for $\alpha_{2}>0$ and $s= \pm 1$, the scalar field reduces by passing the time, as expected. Then, by assuming the simplest generalization of the brane energy density $\left(\rho^{(b)}=W_{0} \rho\right)$ and by choosing some ansatz for the scalar field and the scale factor $\left(\phi=\phi_{0} e^{-\alpha t}, a=a_{0} e^{\beta t}\right.$ and $\left.a=a_{0} t^{\nu}\right)$, we have studied the status of the conservation equation on the brane. Our analysis has shown that just for $\alpha_{2}>0$ and $s=+1$, the right hand side of the energy conservation equation is positive. This means that, for these values of parameters the energy leaks off the brane, as the universe expands. So, just with $\alpha_{2}>0$ and $s=+1$, the model leads to a viable cosmology. Finally, we have studied the late time behavior of the scenario. We have shown that the normal branch of this DGP-inspired model, in the presence of a non-minimally coupled bulk scalar field, realizes a phantom-like behavior, can explain the late time cosmic acceleration and a smooth phantom divide crossing by effective equation of state parameter on the brane.

\section{Acknowledgement}

This work has been supported financially by the Research Council of the Islamic Azad University, Sari Branch, Sari, Iran.

\section{Appendix A}

$$
\begin{gathered}
H^{2}=\frac{1}{\left(-3 \kappa_{5}^{2}-16 \xi^{2} \phi_{0}^{2} \kappa_{5}^{2}+3 \xi \phi_{0}^{2} \kappa_{5}^{2}-24 \xi^{2} \phi_{0}^{2} \kappa_{4}^{2}+24 \xi^{3} \phi_{0}^{4} \kappa_{4}^{2}\right)^{2}}\left\{384 \xi^{2} \phi^{2} \kappa_{4}^{4}+2048 \xi^{4} \phi^{4} \kappa_{4}^{4}\right. \\
+2048 \xi^{6} \phi^{8} \kappa_{4}^{4}-72 \kappa_{4}^{4} \xi^{3} \phi^{6}-4096 \xi^{5} \phi^{6} \kappa_{4}^{4}-1152 \xi^{3} \phi^{4} \kappa_{4}^{4}-384 \kappa_{4}^{4} \xi^{5} \phi^{8}-1152 \xi^{5} \phi^{6} \kappa_{4}^{4} m \\
+48 \xi^{3} \phi^{4} \kappa_{5}^{4} m-48 \xi^{2} \phi^{2} \kappa_{5}^{4} m+576 \xi^{6} \phi^{8} \kappa_{4}^{4} m+576 \xi^{4} \phi^{4} \kappa_{4}^{4} m-256 \xi^{4} \phi^{4} \kappa_{5}^{4} m+108 \kappa_{4}^{4} \xi^{2} \phi^{4} \\
+3 \rho^{(b)} \kappa_{5}^{4} \kappa_{4}^{2}+1152 \xi^{4} \phi^{6} \kappa_{4}^{4}+18 \kappa_{4}^{4} \xi^{4} \phi^{8}-72 \kappa_{4}^{4} \xi \phi^{2}+18 \kappa_{4}^{4}+192 \xi^{4} \phi^{4} \rho^{(b)} \kappa_{5}^{2} \kappa_{4}^{4}-6 \xi \phi^{2} \kappa_{5}^{4} \rho^{(b)} \kappa_{4}^{2} \\
-144 \xi^{3} \phi^{4} \kappa_{4}^{2} m \kappa_{5}^{2}+96 \xi^{4} \phi^{5} \kappa_{4}^{4} g \kappa_{5}^{2}+72 \xi^{2} \phi^{2} \kappa_{4}^{2} m \kappa_{5}^{2}-40 \xi^{3} \phi^{4} \rho^{(b)} \kappa_{5}^{4} \kappa_{4}^{2}-48 \xi^{5} \phi^{7} \kappa_{4}^{4} g \kappa_{5}^{2} \\
-48 \xi^{3} \phi^{3} \kappa_{4}^{4} g \kappa_{5}^{2}+128 \xi^{4} \phi^{4} p \kappa_{5}^{4} \kappa_{4}^{2}+72 \xi^{4} \phi^{6} \kappa_{4}^{2} m \kappa_{5}^{2}-192 \xi^{5} \phi^{6} \kappa_{4}^{4} \rho^{(b)} \kappa_{5}^{2}+3 \xi^{2} \phi^{4} \kappa_{5}^{4} \rho^{(b)} \kappa_{4}^{2} \\
+12 \xi^{2} \phi^{3} \kappa_{4}^{2} g \kappa_{5}^{4}-32 \xi^{3} \phi^{3} \kappa_{5}^{4} \kappa_{4}^{2} g-24 \xi^{3} \phi^{4} p \kappa_{5}^{4} \kappa_{4}^{2}+40 \xi^{2} \phi^{2} \rho^{(b)} \kappa_{5}^{4} \kappa_{4}^{2}-6 \xi \phi \kappa_{4}^{2} g \kappa_{5}^{4}+24 \xi^{4} \phi^{6} \kappa_{4}^{4} \rho^{(b)} \kappa_{5}^{2}
\end{gathered}
$$




$$
\begin{aligned}
& -6 \xi^{3} \phi^{5} \kappa_{4}^{2} g \kappa_{5}^{4}+192 \xi^{4} \phi^{4} \kappa_{4}^{4} p \kappa_{5}^{2}-48 \xi^{3} \phi^{4} \kappa_{4}^{4} \rho^{(b)} \kappa_{5}^{2}+24 \xi^{2} \phi^{2} \kappa_{4}^{4} \rho^{(b)} \kappa_{5}^{2}-192 \xi^{5} \phi^{6} \kappa_{4}^{4} p \kappa_{5}^{2} \\
& +128 \xi^{4} \phi^{4} \rho^{(b)} \kappa^{4} \kappa_{4}^{2}+24 \xi^{2} \phi^{2} p \kappa_{5}^{4} \kappa_{4}^{2}+32 \xi^{4} \phi^{5} \kappa_{4}^{2} g \kappa_{5}^{4} \\
& \pm 2\left[\kappa _ { 4 } ^ { 4 } ( \xi \phi ^ { 2 } - 1 ) ^ { 2 } ( 3 2 \xi ^ { 2 } \phi ^ { 2 } - 3 \xi \phi ^ { 2 } + 3 ) ^ { 2 } \left(192 \xi^{2} \phi^{2} \kappa_{4}^{4}+1024 \xi^{4} \phi^{4} \kappa_{4}^{4}+1024 \xi^{6} \phi^{8} \kappa_{4}^{4}-36 \kappa_{4}^{4} \xi^{3} \phi^{6}\right.\right. \\
& -2048 \xi^{5} \phi^{6} \kappa_{4}^{4}-576 \xi^{3} \phi^{4} \kappa_{4}^{4}-192 \kappa_{4}^{4} \xi^{5} \phi^{8}-96 \kappa_{5}^{4} \mathcal{F} \xi^{3} \phi^{4}+256 \xi^{4} \phi^{4} \kappa_{5}^{4} \mathcal{F}+576 \xi^{6} \phi^{8} \kappa_{4}^{4} \mathcal{F} \\
& +576 \xi^{4} \phi^{4} \kappa_{4}^{4} \mathcal{F}-18 \kappa_{5}^{4} \mathcal{F} \xi \phi^{2}+9 \kappa_{5}^{4} \mathcal{F} \xi^{2} \phi^{4}+96 \kappa_{5}^{4} \mathcal{F} \xi^{2} \phi^{2}-1152 \xi^{5} \phi^{6} \kappa_{4}^{4} \mathcal{F}-1152 \xi^{5} \phi^{6} \kappa_{4}^{4} m \\
& +48 \xi^{3} \phi^{4} \kappa_{5}^{4} m-48 \xi^{2} \phi^{2} \kappa_{5}^{4} m+576 \xi^{6} \phi^{8} \kappa_{4}^{4} m+576 \xi^{4} \phi^{4} \kappa_{4}^{4} m-256 \xi^{4} \phi^{4} \kappa_{5}^{4} m+54 \kappa_{4}^{4} \xi^{2} \phi^{4} \\
& +3 \rho^{(b)} \kappa_{5}^{4} \kappa_{4}^{2}+576 \xi^{4} \phi^{6} \kappa_{4}^{4}+9 \kappa_{4}^{4} \xi^{4} \phi^{8}-36 \kappa_{4}^{4} \xi \phi^{2}+9 \kappa_{4}^{4}+9 \kappa_{5}^{4} \mathcal{F}+192 \xi^{4} \phi^{4} \rho^{(b)} \kappa_{5}^{2} \kappa_{4}^{4}-6 \xi \phi^{2} \kappa_{5}^{4} \rho^{(b)} \kappa_{4}^{2} \\
& -144 \xi^{3} \phi^{4} \kappa_{4}^{2} m \kappa_{5}^{2}+96 \xi^{4} \phi^{5} \kappa_{4}^{4} g \kappa_{5}^{2}+72 \xi^{2} \phi^{2} \kappa_{4}^{2} m \kappa_{5}^{2}-40 \xi^{3} \phi^{4} \rho^{(b)} \kappa_{5}^{4} \kappa_{4}^{2}-48 \xi^{5} \phi^{7} \kappa_{4}^{4} g \kappa_{5}^{2}-48 \xi^{3} \phi^{3} \kappa_{4}^{4} g \kappa_{5}^{2} \\
& -288 \xi^{3} \phi^{4} \kappa_{4}^{2} \kappa_{5}^{2} \mathcal{F}+144 \xi^{2} \phi^{2} \kappa_{4}^{2} \kappa_{5}^{2} \mathcal{F}+128 \xi^{4} \phi^{4} p \kappa_{5}^{4} \kappa_{4}^{2}+72 \xi^{4} \phi^{6} \kappa_{4}^{2} m \kappa_{5}^{2}-192 \xi^{5} \phi^{6} \kappa_{4}^{4} \rho^{(b)} \kappa_{5}^{2} \\
& +3 \xi^{2} \phi^{4} \kappa_{5}^{4} \rho^{(b)} \kappa_{4}^{2}-768 \kappa_{4}^{2} \xi^{5} \phi^{6} \kappa_{5}^{2} \mathcal{F}+144 \xi^{4} \phi^{6} \kappa_{4}^{2} \kappa_{5}^{2} \mathcal{F}+768 \kappa_{4}^{2} \xi^{4} \phi^{4} \kappa_{5}^{2} \mathcal{F}+12 \xi^{2} \phi^{3} \kappa_{4}^{2} g \kappa^{4} \\
& -32 \xi^{3} \phi^{3} \kappa_{5}^{4} \kappa_{4}^{2} g-24 \xi^{3} \phi^{4} p \kappa_{5}^{4} \kappa_{4}^{2}+40 \xi^{2} \phi^{2} \rho^{(b)} \kappa_{5}^{4} \kappa_{4}^{2}-6 \xi \phi \kappa_{4}^{2} g \kappa_{5}^{4}+24 \xi^{4} \phi^{6} \kappa_{4}^{4} \rho^{(b)} \kappa_{5}^{2}-6 \xi^{3} \phi^{5} \kappa_{4}^{2} g \kappa_{5}^{4} \\
& +192 \xi^{4} \phi^{4} \kappa_{4}^{4} p \kappa_{5}^{2}-48 \xi^{3} \phi^{4} \kappa_{4}^{4} \rho^{(b)} \kappa_{5}^{2}+24 \xi^{2} \phi^{2} \kappa_{4}^{4} \rho^{(b)} \kappa_{5}^{2}-192 \xi^{5} \phi^{6} \kappa_{4}^{4} p \kappa_{5}^{2}+128 \xi^{4} \phi^{4} \rho^{(b)} \kappa_{5}^{4} \kappa_{4}^{2} \\
& \left.\left.\left.+24 \xi^{2} \phi^{2} p \kappa_{5}^{4} \kappa_{4}^{2}+32 \xi^{4} \phi^{5} \kappa_{4}^{2} g \kappa_{5}^{4}\right)\right]^{\frac{1}{2}}\right\}_{0}
\end{aligned}
$$

\section{Appendix B}

$$
\begin{aligned}
& \omega_{e f f}=-\frac{1}{3}\left\{\left[-96 \xi^{2} \phi \kappa_{5}^{4} m \dot{\phi}-48 \xi^{2} \phi^{2} \kappa_{5}^{4} \dot{m}+144 \xi^{2} \phi \kappa_{4}^{2} m \kappa_{5}^{2} \dot{\phi}+72 \xi^{2} \phi^{2} \kappa_{4}^{2} \dot{m} \kappa_{5}^{2}-12 \xi \phi \kappa_{5}^{4} \rho^{(b)} \kappa_{4}^{2} \dot{\phi}-\dot{\rho}_{m}\right.\right. \\
& +48 \xi^{2} \phi \kappa_{4}^{4} \rho^{(b)} \kappa_{5}^{2} \dot{\phi}+24 \xi^{2} \phi^{2} \kappa_{4}^{4} \dot{\rho}^{(b)} \kappa_{5}^{2}+48 \xi^{2} \phi p \kappa_{5}^{4} \kappa_{4}^{2} \dot{\phi}+24 \xi^{2} \phi^{2} \dot{p} \kappa_{5}^{4} \kappa_{4}^{2}+36 \xi^{2} \phi^{2} \kappa_{4}^{2} g \kappa_{5}^{4} \dot{\phi}+12 \xi^{2} \phi^{3} \kappa_{4}^{2} \dot{g} \kappa_{5}^{4} \\
& -6 \xi \phi^{2} \kappa_{5}^{4} \dot{\rho}^{(b)} \kappa_{4}^{2}+80 \xi^{2} \phi \rho^{(b)} \kappa_{5}^{4} \kappa_{4}^{2} \dot{\phi}+40 \xi^{2} \phi^{2} \dot{\rho}^{(b)} \kappa_{5}^{4} \kappa_{4}^{2}-6 \xi \dot{\phi} \kappa_{4}^{2} g \kappa_{5}^{4}-6 \xi \phi \kappa_{4}^{2} \dot{g} \kappa_{5}^{4}+12 \xi^{2} \phi^{3} \kappa_{5}^{4} \rho^{(b)} \kappa_{4}^{2} \dot{\phi} \\
& +3 \xi^{2} \phi^{4} \kappa_{5}^{4} \dot{\rho}^{(b)} \kappa_{4}^{2}+3 \dot{\rho}^{(b)} \kappa_{5}^{4} \kappa_{4}^{2}+432 \kappa_{4}^{4} \xi^{2} \phi^{3} \dot{\phi}+768 \xi^{2} \phi \kappa_{4}^{4} \dot{\phi}-144 \kappa_{4}^{4} \xi \phi \dot{\phi}-3 \kappa_{4}^{8}\left(3 \dot{\rho}^{(b)} \kappa_{5}^{4} \kappa_{4}^{2}+48 \xi^{2} \phi \kappa_{4}^{4} \rho^{(b)} \kappa_{5}^{2} \dot{\phi}\right. \\
& +48 \xi^{2} \phi p \kappa_{5}^{4} \kappa_{4}^{2} \dot{\phi}+108 \xi^{2} \phi^{2} \kappa_{4}^{2} g \kappa_{5}^{4} \dot{\phi}+208 \xi^{2} \phi \rho^{(b)} \kappa_{5}^{4} \kappa_{4}^{2} \dot{\phi}+180 \xi^{2} \phi^{3} \kappa_{5}^{4} \rho^{(b)} \kappa_{4}^{2} \dot{\phi}+288 \xi^{2} \phi \kappa_{4}^{2} \kappa_{5}^{2} \mathcal{F} \dot{\phi}+9 \kappa_{5}^{4} \dot{\mathcal{F}} \\
& -12 \xi \phi \kappa_{4}^{2} g \kappa_{5}^{4} \dot{\phi}-96 \xi^{2} \phi \kappa_{5}^{4} m \dot{\phi}+72 \xi^{2} \phi^{2} \kappa_{4}^{2} \dot{m} \kappa_{5}^{2}-18 \xi \phi^{2} \kappa_{5}^{4} \dot{\rho}^{(b)} \kappa_{4}^{2}+24 \xi^{2} \phi^{2} \kappa_{4}^{4} \dot{\rho}^{(b)} \kappa_{5}^{2}+24 \xi^{2} \phi^{2} \dot{p} \kappa_{5}^{4} \kappa_{4}^{2} \\
& +36 \xi^{2} \phi^{3} \kappa_{4}^{2} \dot{g} \kappa_{5}^{4}+104 \xi^{2} \phi^{2} \dot{\rho}^{(b)} \kappa_{5}^{4} \kappa_{4}^{2}+45 \xi^{2} \phi^{4} \kappa_{5}^{4} \dot{\rho}^{(b)} \kappa_{4}^{2}-6 \xi \phi^{2} \kappa_{4}^{2} \dot{g} \kappa_{5}^{4}+144 \xi^{2} \phi^{2} \kappa_{4}^{2} \kappa_{5}^{2} \dot{\mathcal{F}}+576 \kappa_{5}^{4} \mathcal{F} \xi^{2} \phi \dot{\phi} \\
& +540 \kappa_{5}^{4} \mathcal{F} \xi^{2} \phi^{3} \dot{\phi}-108 \kappa_{5}^{4} \mathcal{F} \xi \phi \dot{\phi}-36 \xi \phi \kappa_{5}^{4} \rho^{(b)} \kappa_{4}^{2} \dot{\phi}+144 \xi^{2} \phi \kappa_{4}^{2} m \kappa_{5}^{2} \dot{\phi}-48 \xi^{2} \phi^{2} \kappa_{5}^{4} \dot{m}+1008 \kappa_{4}^{4} \xi^{2} \phi^{3} \dot{\phi} \\
& \left.-144 \kappa_{4}^{4} \xi \phi \dot{\phi}+288 \kappa_{5}^{4} \dot{\mathcal{F}} \xi^{2} \phi^{2}+135 \kappa_{5}^{4} \dot{\mathcal{F}} \xi^{2} \phi^{4}-54 \kappa_{5}^{4} \dot{\mathcal{F}} \xi \phi^{2}\right)\left(24 \xi^{2} \phi^{2} \kappa_{4}^{4} \rho^{(b)} \kappa_{5}^{2}-72 \kappa_{4}^{4} \xi \phi^{2}+9 \kappa_{4}^{4}\right. \\
& +252 \kappa_{4}^{4} \xi^{2} \phi^{4}+72 \xi^{2} \phi^{2} \kappa_{4}^{2} m \kappa_{5}^{2}-18 \xi \phi^{2} \kappa_{5}^{4} \rho^{(b)} \kappa_{4}^{2}-6 \xi \phi^{2} \kappa_{4}^{2} g \kappa_{5}^{4}+24 \xi^{2} \phi^{2} p \kappa_{5}^{4} \kappa_{4}^{2}+36 \xi^{2} \phi^{3} \kappa_{4}^{2} g \kappa_{5}^{4} \\
& +104 \xi^{2} \phi^{2} \rho^{(b)} \kappa_{5}^{4} \kappa_{4}^{2}+45 \xi^{2} \phi^{4} \kappa_{5}^{4} \rho^{(b)} \kappa_{4}^{2}+144 \xi^{2} \phi^{2} \kappa_{4}^{2} \kappa_{5}^{2} \mathcal{F}+3 \rho^{(b)} \kappa_{5}^{4} \kappa_{4}^{2}+9 \kappa_{5}^{4} \mathcal{F}-48 \xi^{2} \phi^{2} \kappa_{5}^{4} m+288 \kappa_{5}^{4} \mathcal{F} \xi^{2} \phi^{2}
\end{aligned}
$$




$$
\begin{gathered}
\left.\left.+135 \kappa_{5}^{4} \mathcal{F} \xi^{2} \phi^{4}-54 \kappa_{5}^{4} \mathcal{F} \xi \phi^{2}\right)^{-1 / 2}\right]\left(3 \kappa_{5}^{2}+16 \xi^{2} \phi^{2} \kappa_{5}^{2}-3 \xi \phi^{2} \kappa_{5}^{2}+24 \xi^{2} \phi^{2} \kappa_{4}^{2}\right)^{-4} H^{-3} \\
\left.-2 H^{-1}\left(3 \kappa_{5}^{2}+16 \xi^{2} \phi^{2} \kappa_{5}^{2}-3 \xi \phi^{2} \kappa_{5}^{2}+24 \xi^{2} \phi^{2} \kappa_{4}^{2}\right)^{-1}\left(32 \xi^{2} \phi \kappa_{5}^{2} \dot{\phi}-6 \xi \phi \kappa_{5}^{2} \dot{\phi}+48 \xi^{2} \phi \kappa_{4}^{2} \dot{\phi}\right)\right\}_{0}-1
\end{gathered}
$$

\section{Appendix C}

$$
\begin{aligned}
& q=-1-\frac{1}{2 H}\left\{\left[768 \kappa_{4}^{4} \xi^{2} \phi \dot{\phi}-144 \kappa_{4}^{4} \xi \phi \dot{\phi}+432 \kappa_{4}^{4} \xi^{2} \phi^{3} \dot{\phi}-96 \xi^{2} \phi \kappa_{5}^{4} m \dot{\phi}-48 \xi^{2} \phi^{2} \kappa_{5}^{4} \dot{m}-6 \xi \phi \kappa_{4}^{2} \dot{g} \kappa_{5}^{4}\right.\right. \\
& -12 \xi \phi \kappa_{5}^{4} \rho \kappa_{4}^{2} \dot{\phi}-6 \xi \phi^{2} \kappa_{5}^{4} \dot{\rho}^{(b)} \kappa_{4}^{2}-6 \xi \dot{\phi} \kappa_{4}^{2} g \kappa_{5}^{4}+80 \xi^{2} \phi \rho \kappa_{5}^{4} \kappa_{4}^{2} \dot{\phi}+40 \xi^{2} \phi^{2} \dot{\rho}^{(b)} \kappa_{5}^{4} \kappa_{4}^{2}+48 \kappa_{4}^{4} \xi^{2} \phi \rho \kappa_{5}^{2} \dot{\phi} \\
& +24 \kappa_{4}^{4} \xi^{2} \phi^{2} \dot{\rho}^{(b)} \kappa_{5}^{2}+144 \xi^{2} \phi^{2} \kappa_{4}^{2} m \kappa_{5}^{2} \dot{\phi}+72 \xi^{2} \phi^{2} \kappa_{4}^{2} \dot{m} \kappa_{5}^{2}+48 \xi^{2} \phi p \kappa_{5}^{4} \kappa_{4}^{2} \dot{\phi}+24 \xi^{2} \phi^{2} \dot{p}^{(b)} \kappa_{5}^{4} \kappa_{4}^{2} \\
& +12 \xi^{2} \phi^{3} \kappa_{5}^{4} \rho \kappa_{4}^{2} \dot{\phi}+3 \xi^{2} \phi^{4} \kappa_{5}^{4} \dot{\rho}^{(b)} \kappa_{4}^{2}+3 \dot{\rho}^{(b)} \kappa_{5}^{4} \kappa_{4}^{2}-3\left\{9 \kappa_{5}^{4} \dot{\mathcal{F}} \kappa_{4}^{4}+3 \kappa_{4}^{6} \dot{\rho}^{(b)} \kappa_{5}^{4}+104 \kappa_{4}^{6} \xi^{2} \phi^{2} \dot{\rho}^{(b)} \kappa_{5}^{4}\right. \\
& +45 \kappa_{4}^{6} \xi^{2} \phi^{4} \kappa_{5}^{4} \dot{\rho}^{(b)}+36 \kappa_{4}^{6} \xi^{2} \phi^{3} \dot{g} \kappa_{5}^{4}-48 \kappa_{4}^{4} \xi^{2} \phi^{2} \kappa_{5}^{4} \dot{m}-6 \kappa_{4}^{6} \xi \dot{\phi} g \kappa_{5}^{4}-6 \kappa_{4}^{6} \xi \phi \dot{g} \kappa_{5}^{4}+24 \kappa_{4}^{8} \xi^{2} \phi^{2} \dot{\rho}^{(b)} \kappa_{5}^{2} \\
& +72 \kappa_{4}^{6} \xi^{2} \phi^{2} \dot{m} \kappa_{5}^{2}+24 \kappa_{4}^{6} \xi^{2} \phi^{2} \dot{p}^{(b)} \kappa_{5}^{4}+144 \xi^{2} \phi^{2} \kappa_{5}^{2} \kappa_{4}^{6} \dot{\mathcal{F}}+288 \kappa_{5}^{4} \dot{\mathcal{F}} \kappa_{4}^{4} \xi^{4} \phi^{6}-54 \kappa_{5}^{4} \dot{\mathcal{F}} k a p p a_{4}^{4} \xi \phi^{2} \\
& +135 \kappa_{5}^{4} \dot{\mathcal{F}} \kappa_{4}^{4} \xi^{2} \phi^{4}+208 \kappa_{4}^{6} \xi^{2} \phi \rho \kappa_{5}^{4} \dot{\phi}+180 \kappa_{4}^{6} \xi^{2} \phi^{3} \kappa_{5}^{4} \rho \dot{\phi}+108 \kappa_{4}^{6} \xi^{2} \phi^{2} g \kappa_{5}^{4} \dot{\phi}-96 \kappa_{4}^{4} \xi^{2} \phi \kappa_{5}^{4} m \dot{\phi} \\
& +48 \kappa_{4}^{8} \xi^{2} \phi \rho \kappa_{5}^{2} \dot{\phi}+144 \kappa_{4}^{6} \xi^{2} \phi m \kappa_{5}^{2} \dot{\phi}+48 \kappa_{4}^{6} \xi^{2} \phi p \kappa_{5}^{4} \dot{\phi}+288 \xi^{2} \phi \kappa_{5}^{2} \kappa_{4}^{6} \mathcal{F} \dot{\phi}+1728 \kappa_{5}^{4} \mathcal{F} \kappa_{4}^{4} \xi^{4} \phi^{5} \dot{\phi} \\
& \left.-108 \kappa_{5}^{4} \mathcal{F} \kappa_{4}^{4} \xi \phi \dot{\phi}+540 \kappa_{5}^{4} \mathcal{F} \kappa_{4}^{4} \xi^{2} \phi^{3} \dot{\phi}+1008 \kappa_{4}^{8} \xi^{2} \phi^{3} \dot{\phi}-144 \kappa_{4}^{8} \xi \phi \dot{\phi}+768 \kappa_{4}^{8} \xi^{2} \phi \dot{\phi}\right\}\left\{\left(9 \kappa_{5}^{4} \mathcal{F} \kappa_{4}^{4}\right.\right. \\
& +384 \kappa_{4}^{8} \xi^{2} \phi^{2}+252 \kappa_{4}^{8} \xi^{2} \phi^{4}-72 \kappa_{4}^{8} \xi \phi^{2}+3 \kappa_{4}^{6} \rho \kappa_{5}^{4}+104 \kappa_{4}^{6} \xi^{2} \phi^{2} \rho \kappa_{5}^{4}+45 \kappa_{4}^{6} \xi^{2} \phi^{4} \kappa_{5}^{4} \rho-6 \kappa_{4}^{6} \xi \phi g \kappa_{5}^{4} \\
& +36 \kappa_{4}^{6} \xi^{2} \phi^{3} g \kappa_{5}^{4}+9 \kappa_{4}^{8}-48 \kappa_{4}^{4} \xi^{2} \phi^{2} \kappa_{5}^{4} m+24 \kappa_{4}^{8} \xi^{2} \phi^{2} \rho \kappa_{5}^{2}+72 \kappa_{4}^{6} \xi^{2} \phi^{2} m \kappa_{5}^{2}+24 \kappa_{4}^{6} \xi^{2} \phi^{2} p \kappa_{5}^{4} \\
& \left.\left.\left.+144 \xi^{2} \phi^{2} \kappa_{5}^{2} \kappa_{4}^{6} \mathcal{F}+288 \kappa_{5}^{4} \mathcal{F} \kappa_{4}^{4} \xi^{4} \phi^{6}-54 \kappa_{5}^{4} \mathcal{F} \kappa_{4}^{4} \xi \phi^{2}+135 \kappa_{5}^{4} \mathcal{F} \kappa_{4}^{4} \xi^{2} \phi^{4}\right)^{\frac{1}{2}}\right\}^{-1}\right]\left(144 \xi^{2} \phi^{2} \kappa_{5}^{2} \kappa_{4}^{2}+9 \kappa_{5}^{4}\right. \\
& \left.\left.+96 \xi^{2} \phi^{2} \kappa_{5}^{4}+9 \xi^{2} \phi^{4} \kappa_{5}^{4}-18 \xi \phi^{2} \kappa_{5}^{4}\right)^{-1}-H^{2}\left(288 \xi^{2} \phi \kappa_{5}^{2} \kappa_{4}^{2} \dot{\phi}+192 \xi^{2} \phi \kappa_{5}^{4} \dot{\phi}+36 \xi^{2} \phi^{3} \kappa_{5}^{4} \dot{\phi}-36 \xi \phi \kappa_{5}^{4} \dot{\phi}\right)\right\}_{0}
\end{aligned}
$$

\section{References}

[1] A. G. Riess et al., Astron. J. 116 (1998) 1009

S. J. Perlmutter et al., Astrophys. J. 517 (1999) 565

A. G. Riess et al., Astrophys. J. 607 (2004) 665

P. Astier et al., Astron. Astrophys. 447 (2006) 31

W. M. Wood-Vasey et al., Astrophys. J. 666 (2007) 694-715

D. N. Spergel et al., Astrophys. J. Suppl 170 (2007) 377

G. Hinshaw et al., Astrophys. J. Suppl , 288 (2007) 170 
M. Colless et al, Mon. Not. R. Astron. Soc. 328 (2001) 1039

M. Tegmark et al., Phys. Rev. D 69 (2004) 103501

S. Cole et al., Mon. Not. R. Astron. Soc. 362 (2005) 505

V. Springel, C. S. Frenk and S. M. D. White, Nature (London) 440 (2006) 1137

S. P. Boughn and R. G. Crittenden, Nature 427 (2004) 24

J. D. McEwen et al., Mon. Not. R. Astron. Soc. 376 (2007) 1211

E. Komatsu et al. [WMAP Collaboration], Astrophys. J. Suppl. 180 (2009) 330.

[2] T. Padmanabhan, Phys. Rept. 380 (2003) 235

S. M. Carroll, Living Rev. Rel. 4 (2001) 1.

[3] E. J. Copeland, M. Sami and S. Tsujikawa, Int. J. Mod. Phys. D 15 (2006) 1753

S. Nojiri and S. D. Odintsov, Phys. Rev. D 70 (2004) 103522

R. R. Caldwell, Phys. Lett. B 545 (2002) 23-29

F. Piazza and S. Tsujikawa, JCAP 0407 (2004) 004

H. Wei and R. G. Cai, Phys. Rev. D 73 (2006) 083002

A. Vikman, Phys. Rev. D 71 (2005) 023515

A. Anisimov, E. Babichev and A. Vikman, JCAP 0506 (2005)006

B. Wang, Y.G. Gong and E. Abdalla, Phys. Lett. B 624 (2005) 141

S. Nojiri and S. D. Odintsov, [arXiv:hep-th/0506212]

S. Nojiri, S. D. Odintsov and S. Tsujikawa, [arXiv:hep-th/0501025]

E. Elizalde, S. Nojiri, S. D. Odintsov and P. Wang, Phys. Rev. D 71 (2005) 103504

W. Zhao and Y. Zhang, Phys. Rev.D 73 (2006)123509

U. Alam, V. Sahni and A. A. Starobinsky, JCAP 06 (2004) 008

S. Nesseris and L. Perivolaropoulos, Phys. Rev. D 72 (2005) 123519

M. Libanov, E. Papantonopoulos, V. Rubakov, M. Sami and S. Tsujikawa, JCAP 0708 (2007) 010

R. J. Scherrer and A. A. Sen, Phys. Rev. D 78 (2008) 067303

F. Briscese, E. Elizalde, S. Nojiri and S. D. Odintsov, Phys. Lett. B 646 (2007) 105-111

M. Sami, [arXiv:0901.0756]

M. Sami, Curr. Sci. 97 (2009) 887

M. Sami, M. Shahalam, M. Skugoreva and A. Toporensky, [arXiv:1207.6691]

G. Caldera-Cabral, R. Maartens and L. A. Urena-Lopez, Phys. Rev. D 79 ( 2009) 063518

V. Sahni and A. Starobinsky, Int. J. Mod. Phys. D 15 (2006) 2105

V. Sahni, Lect. Notes Phys. 653 (2004) 141

Y. -F. Cai, E. N. Saridakis, M. R. Setare and J. -Q. Xia, Phys. Rept. 493 (2010) 1-60

H. Zhang, [arXiv:0909.3013].

[4] S. Capozziello, V. F. Cardone, S. Carloni and A. Troisi, Int. J. Mod. Phys. D 12 (2003) 1969

S. Nojiri and S. D. Odintsov, Int. J. Geom. Meth. Mod. Phys. 4 (2007) 115

T. P. Sotiriou and V. Faraoni, Rev. Mod. Phys. 82 (2010) 451

S. Nojiri and S. D. Odintsov, Gen. Relat. Gravit. 36 (2004) 1765 
S. Nojiri and S. D. Odintsov, Phys. Rev. D68 (2003) 123512

S. Nojiri and S. D. Odintsov, Class. Quantum Grav. 22 (2005) L35

S. Nojiri and S. D. Odintsov, Phys. Rev. D 74 (2006) 086009

K. Bamba, S. Nojiri and S. D. Odintsov, Phys. Rev. D. 79 (2009) 083014

S. Nojiri and S. D. Odintsov, Phys. Rev. D. 78 (2008) 046006

K. Bamba, S. Nojiri and S. D. Odintsov, JCAP 0810 (2008) 045

S. M. Carroll, V. Duvvuri, M. Trodden and M. S. Turner, Phys. Rev. D70 (2004) 043528

L. Amendola, D. Polarski and S. Tsujikawa Phys. Rev. Lett. 98 (2007) 131302

I. Sawicki and W. Hu, Phys. Rev. D 75 (2007) 127502

W. Hu and I. Sawicki, Phys. Rev. D 75 (2007) 044004

S. Nojiri and S. D. Odintsov, AIP Conf. Proc. 1115 (2009) 212-217

S. Nojiri and S. D. Odintsov, Phys. Rept. 505 (2011) 59

A. De Felice and S. Tsujikawa, Living Rev. Rel. 13 (2010) 3

S. Tsujikawa, Lect. Notes Phys. 800 (2010) 99.

[5] N. Arkani-Hamed, S. Dimopoulos, G. Dvali, Phys. Lett. B 429 (1998) 263

N. Arkani-Hamed, S. Dimopoulos, G. Dvali, Phys. Rev. D 59 (1999) 086004

I. Antoniadis, N. Arkani-Hamed, S. Dimopoulos and G. Dvali, Phys. Lett. B 436 (1998) 257.

[6] L. Randall, R. Sundrum, Phys. Rev. Lett. 83 (1999) 3370

L. Randall, R. Sundrum, Phys. Rev. Lett. 83 (1999) 4690.

[7] G. Dvali, G. Gabadadze and M. Porrati, Phys. Lett. B 485 (2000) 208

G. Dvali and G. Gabadadze, Phys. Rev. D 63 (2001) 065007 G. Dvali

G. Dvali, G. Gabadadze, M. Kolanovic and F. Nitti, Phys. Rev. D, 64, (2001) 084004.

[8] A. Lue, Phys. Rept. 423 (2006) 1

[9] R. Ferraro and F. Fiorini, Phys. Rev. D 75 (2007)084031

R. Ferraro and F. Fiorini, Phys. Rev. D 78 (2008)124019

P. Wu and H. W. Yu, Eur. Phys. J. C 71 (2011) 1552

R. Zheng and Q. G. Huang, JCAP 1103 (2011) 002

K. Bamba, C. Q. Geng, C. C. Lee and L. W. Luo, JCAP 1101 (2011) 021

S. H. Chen, J. B. Dent, S. Dutta and E. N. Saridakis, Phys. Rev. D 83 (2011) 023508

J. B. Dent, S. Dutta and E. N. Saridakis, JCAP 1101 (2011) 009

R. Ferraro and F. Fiorini, Phys. Lett. B 702 (2011) 75

C. Q. Geng, C. C. Lee, E. N. Saridakis, Y. P. Wu, Phys. Lett. B 704 (2011) 384

S. Capozziello, V. F. Cardone, H. Farajollahi and A. Ravanpak, Phys. Rev. D 84 (2011) 043527

K. Karami and A. Abdolmaleki, JCAP 04 (2012) 007

M. Li, R. -X. Miao, Y. -G. Miao, JHEP 1107 (2011) 108

R. -X. Miao, M. Li and Y. -G. Miao, JCAP 11 (2011) 033

A. Behboodi, S. Akhshabi and K. Nozari, [arXiv:1205.4570]. 
[10] K. Koyama, Class. Quantum Grav. 24 (2007) R231

C. de Rham and A. J. Tolley, JCAP 0607 (2006) 004

M. A. Luty, M. Porrati and R. Rattazzi, JHEP 0309 (2003) 029.

[11] V. Sahni and Y. Shtanov, JCAP 0311 (2003) 014

V. Sahni [arXiv:astro-ph/0502032]

A. Lue and G. D. Starkman, Phys. Rev. D 70 (2004) 101501

L. P. Chimento, R. Lazkoz, R. Maartens and I. Quiros, JCAP 0609 (2006) 004

R. Lazkoz, R. Maartens and E. Majerotto, Phys. Rev. D74 (2006) 083510

R. Maartens and E. Majerotto, Phys. Rev. D 74 (2006) 023004

M. Bouhmadi-Lopez, Nucl. Phys. B 797 (2008) 78

M. Bouhmadi-Lopez and A. Ferrera, JCAP 0810 (2008) 011.

[12] K. Nozari and M. Pourghasemi, JCAP 10 (2008) 044

M. Bouhmadi-Lopez and P. Vargas Moniz, Phys. Rev. D 78 (2008) 084019

M. Bouhmadi-Lopez, Y. Tavakoli and P. Vargas Moniz, JCAP 1004 (2010) 016

M. Bouhmadi-Lopez and L. Chimento, Phys. Rev. D 82 (2010) 103506

M. Bouhamdi-Lopez, JCAP 0911 (2009) 011

K. Nozari and N. Rashidi, JCAP 0909 (2009) 014

K. Nozari and N. Rashidi, Int. J. Thoer. Phys. 48 (2009) 2800

K. Nozari and N. Rashidi, Int. J. Mod. Phys. D 19 (2010) 219

K. Nozari and T. Azizi, Phys. Lett. B 680 (2009) 205

K. Nozari and F. Kiani, JCAP 0907 (2009) 010

K. Nozari and N. Rashidi, Phys. Rev. D 86 (2012) 043505

K. Nozari et al., Class. Quantum Grav. 29 (2012) 175002.

[13] P. Kanti, K. A. Olive and M. Pospelov, Phys. Lett. B 481 (2000) 386

E. E. Flanagan, S. -H. Henry Tye and I. Wasserman, Phys. Lett. B522 (2001) 155

D. Langlois and M. Rodriguez-Martinez, Phys. Rev. D 64 (2001) 123507

K. E. Kunze and M. A. Vazquez-Mozo, Phys. Rev. D 65 (2002) 044002

S. C. Davis, JHEP 0203 (2002) 058

M. Parry and S. Pichler, JCAP 0411 (2004) 005

C. Bogdanos, A. Dimitriadis and K. Tamvakis, Class. Quant. Grav. 24 (2007) 3701

M. Heydari-Fard and H. R. Sepangi, JCAP 0901 (2009) 034

R. Ansari and P. K. Suresh, JCAP 0709 (2007) 021

J. M. Cline and H. Firouzjahi, Phys. Rev. D 64 (2001) 023505

S. Kobayashi and K. Koyama, JHEP 0212 (2002) 056

Y. Himemoto and M. Sasaki, Phys. Rev. D 63 (2001) 044015

R. N. Mohapatra, A. Prez-Lorenzana and C. A. de S. Pires, Phys. Rev. D 62 (2000) 105030

Y. Himemoto and M. Sasaki, Prog. Theor. Phys. Suppl. 148 (2003) 235

J. Yokoyama and Y. Himemoto, Phys. Rev. D 64 (2001) 083511.

[14] K.Nozari and N. Rashidi, Astrophys. Space. Sci. 338 (2012) 363. 
[15] K. Nozari and N. Rashidi, Astropart. Phys. 35 (2012) 828.

[16] V. Faraoni, Phys. Rev. D 53 (1996) 6813

V. Faraoni, Phys. Rev. D 62 (2000) 023504

V. Faraoni and E. Gunzig, Int.J.Theor.Phys. 38 (1999) 217.

[17] K. Nozari, Phys. Lett. B 652 (2007) 159

K. Nozari, JCAP 0709 (2007) 003.

[18] J. Saavedra and Y. Vasquez, JCAP 04 (2009) 013

K. Atazadeh and H. R. Sepangi, Phys. Lett. B 643 (2006) 76

K. Atazadeh and H. R. Sepangi, JCAP 01 (2009) 006

A. Borzou, H. R. Sepangi, S. Shahidi and R. Yousefi, EPL 88 (2009) 29001. M. Bouhmadi-Lopez, JCAP 0911 (2009) 011.

M. Bouhmadi-Lopez, S. Capozziello, V. F. Cardone, Phys. Rev. D 82 (2010) 103526.

[19] P. Binetruy, C. Deffayet and D. Langlois, Nuclear Physics B 565 (2000) 269.

[20] P. Binetruy, C. Deffayet, U. Ellwanger and D. Langlois, Phys. Lett. B 477 (2000) 285.

[21] S. C. Davis, JHEP 0203 (2002) 054.

[22] J. Sollerman et al., Astrophys. J. 703 (2009) 1374, [arXiv:0908.4276]. 